\title{
Special solutions to a non-linear coarsening model with local interactions
}

\author{
Constantin Eichenberg \\ Institute for Applied Mathematics, University of Bonn \\ Endenicher Allee 60, 53115 Bonn, Germany \\ eichenberg@iam.uni-bonn.de
}

March 7, 2018

\begin{abstract}
We consider a class of mass transfer models on a one-dimensional lattice with nearest-neighbour interactions. The evolution is given by the backward parabolic equation $\partial_{t} x=-\frac{\beta}{\beta \mid} \Delta x^{\beta}$, with $\beta$ in the fast diffusion regime $(-\infty, 0) \cup(0,1]$. Sites with mass zero are deleted from the system, which leads to a coarsening of the mass distribution. The rate of coarsening suggested by scaling is $t^{\frac{1}{1-\beta}}$ if $\beta \neq 1$ and exponential if $\beta=1$. We prove that such solutions actually exist by an analysis of the time-reversed evolution. In particular we establish positivity estimates and long-time equilibrium properties for discrete parabolic equations with initial data in $\ell_{+}^{\infty}(\mathbb{Z})$.
\end{abstract}

\section{Contents}

1 Introduction

2 Statement of results 4

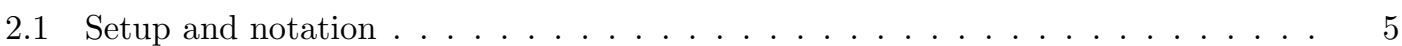

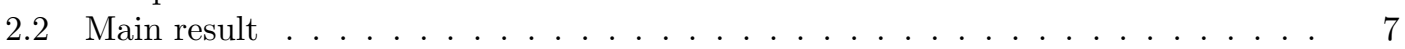

3 Construction of solutions $\mathbf{1 0}$

3.1 Insertion of particles . . . . . . . . . . . . . . . . . . . . . .

3.2 Back-in-time construction . . . . . . . . . . . . . . . . 11

4 Proof of the main result 13

4.1 Step 1: Average modification by particle insertion . . . . . . . . . . . 13

4.2 Step 2: Estimate for the backward equation . . . . . . . . . . . . . 16

4.3 Step 3: Approximating sequence . . . . . . . . . . . . . . . . . . . . . 19

4.4 Step 4: Passage to the limit . . . . . . . . . . . . . . . . 21 
A Appendix

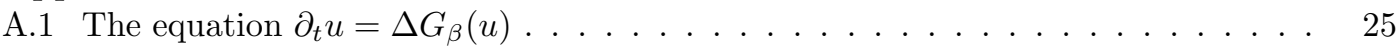

A.2 Existence of solutions for $\beta<0 \ldots \ldots \ldots \ldots \ldots \ldots$

A.3 Harnack-type inequality for $0<\beta \leq 1 \ldots \ldots \ldots \ldots \ldots$. . . . . . . . . 32

A.4 Nash-Aronson estimates and Hölder continuity . . . . . . . . . . . . . 36

\section{Introduction}

Discrete mass transfer models with local interactions have been studied by several authors in different contexts. They have several applications in physics such as the growth and coarsening of sand ripples in [HK02 or the clustering in granular gases vvL01, while also serving as approximations or toy models for more complex coarsening scenarios such as the evolution of droplets in dewetting films GW03, GW05 and grain growth HNO04. If the mass transport between sites is symmetric, the evolution of such systems in one dimension is governed by an infinite system of ODEs,

$$
\frac{\mathrm{d}}{\mathrm{d} t} x(t, k)=F(x(t, k-1))-2 F(x(t, k))+F(x(t, k+1)),
$$

where the right hand side represents the net mass flux at a site $k$ which receives and transfers mass from its neighbours at rates controlled by the flux function $F$. This system can also be interpreted as the spatially discrete non-linear PDE $\partial_{t} x=\Delta F(x)$. The monotonicity properties of $F$ are crucial for the qualitative behaviour of solutions and depend on the application, as an increasing flux function will lead to mass diffusion and a decreasing flux function will lead to aggregation and coarsening. A combination of both is also possible, for example in models that were investigated in [ES08, EG09].

In this paper we are interested in the coarsening model proposed in [HNV16], with flux function

$$
F(x)=F_{\beta}(x)=-\frac{\beta}{|\beta|} x^{\beta},
$$

where $-\infty<\beta<0$ or $0<\beta \leq 1$. This largely resembles the sand ripple scenario [HK02, although we will refer to the lattice points as particles from now on. Distinctive features of the model are the infinite number of particles and the vanishing rule: Particles that reach mass zero are deleted from the system and the remaining particles are relabeled accordingly. This way small particles vanish from the system while transferring their mass to the rest of the system, which leads to a growth of the average particle size and an overall coarsening of the system.

With this particular choice of the flux function (except when $\beta=1$ ), the equation has an invariant scaling: If $x=x(t, k)$ is a solution, then 


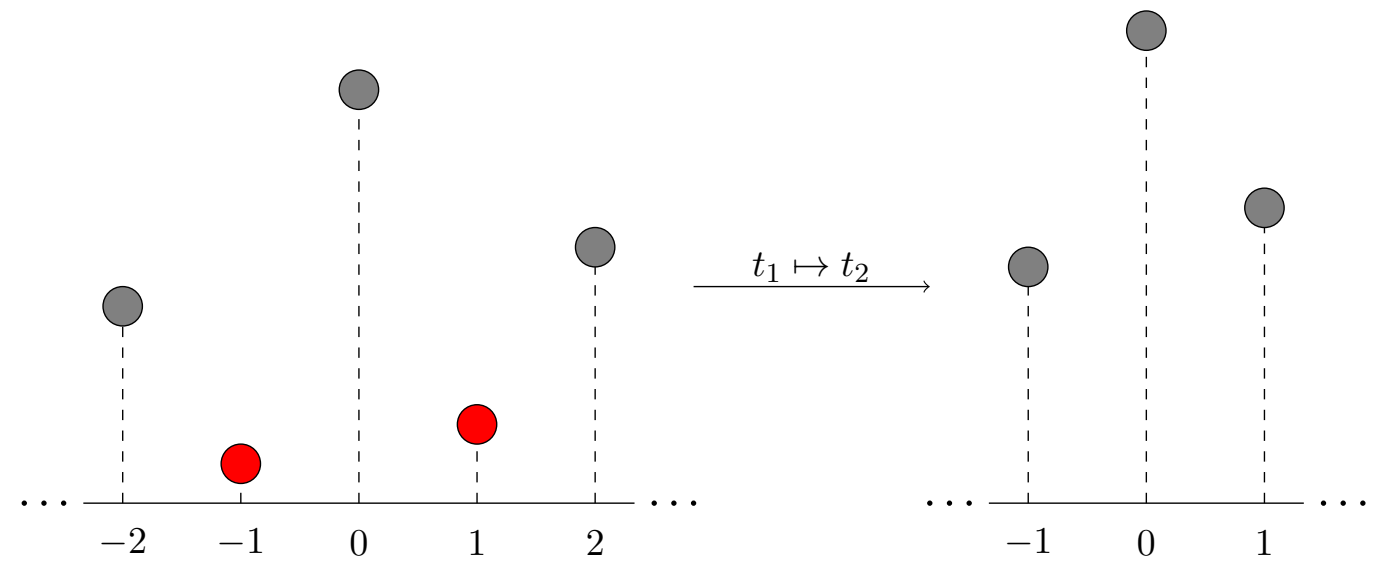

Figure 1: Small particles vanish at $t_{2}$ and the average mass increases.

$$
x_{\lambda}(t, k)=\lambda^{\frac{1}{\beta-1}} x(\lambda t, k)
$$

is another solution. Thus, if $\langle x\rangle$ denotes a suitable lenght-scale, we expect that

$$
\langle x\rangle \sim t^{\frac{1}{1-\beta}} .
$$

In the case $\beta=1$ the mean-field analysis in [HK02] indicates that $\langle x\rangle \sim \exp (\lambda t)$, where $\lambda$ is not universal but depends on the initial distribution.

The problem in the mathematical analysis of such models is to rigorously establish such coarsening rates. The method of Kohn and Otto KO02 has proved very useful in several situations to obtain upper bounds. Here, $\langle x\rangle$ is usually some negative Sobolev norm. In our setting however, their technique is not obviously applicable. Although the system is formally an $H^{-1}$ gradient flow, the corresponding energy is infinite due to the presence of infinitely many particles. Additionally, the vanishing and relabeling of particles is problematic in this context. Still, the simple structure of our model enables us to apply more elementary arguments to derive upper bounds: For positive $\beta$, the right hand side of equation (1.1) can be estimated to obtain

$$
\dot{x} \leq 2 x^{\beta},
$$

which can be integrated to yield the desired bound in the $\ell^{\infty}$-norm. For negative $\beta$ the equation gives $\dot{x} \geq-2 x^{\beta}$, which can be used to derive a weak upper bound, see Proposition 2.4 in HNV16. Furthermore, the numerical simulations and heuristics in [HNV16] demonstrate that single particles can grow linearly (thus faster than the scaling law) in 


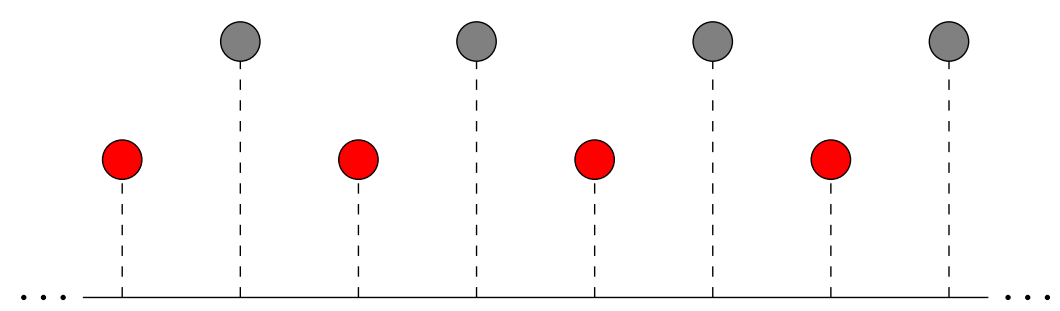

Figure 2: After the smaller particles have vanished, the configuration is constant.

time, showing that an $\ell^{\infty}$-bound cannot be expected in this case.

On the other hand, not much is known about the validity of lower bounds. As will be demonstrated below, there are many non-constant initial configurations which become stationary after a finite time due to the vanishing of particles. An easy example for this is a 2-periodic configuration of large and small particles. During the evolution, the large particles grow and the small particles shrink until disappearing at the same time, at which all large particles will be left with the same size and the evolution stops.

The problem of classifying all initial data for which some form of a lower coarsening bound holds is completely open. The main result of this work is the existence of initial data and corresponding solutions with scale-characteristic coarsening rates, where $\langle x\rangle$ is a suitable average of the configuration, see Theorem 2.2. Our general ansatz is to reverse time, which transforms the equation into a non-linear discrete parabolic equation which behaves much better and can be analysed by means of Harnack-type positivity estimates (see [BV06]) and parabolic regularity theory (see [Nas58] for the continuum theory and GOS01] for the discrete analogue). It should be mentioned that the solutions that we construct coarsen in a very organised manner, whereas numerical simulations and heuristics that were done in [HNV16] indicate that the generic coarsening behaviour is more disorganised. Nevertheless we believe that this result is useful as a first step to a better understanding of the coarsening dynamics of this model. In particular, our methods imply the instability of constant configurations in a rather strong sense, see Corollary 2.4.

The rest of the paper is organised as follows: In Section 2 we give a precise description of the model and our main result. A general technique for the construction of solutions is presented in Section 3, while the main result is proved in Section 4. We postpone the proofs of some necessary technical results such as existence of solutions for the time-reversed setting, Harnack inequalities and discrete Nash-Aronson estimates to the appendix.

\section{Statement of results}

We collect some definitions and introduce appropriate notation to give a rigorous description of our setting. We largely follow [HNV16], where this model was first introduced in 
a mathematical context. Then we state our main result and give a short outline of the proof.

\subsection{Setup and notation}

We consider a discrete infinite number of particles with non-negative mass on a onedimensional lattice. That means each configuration is an element of the space

$$
\ell_{+}^{\infty}(\mathbb{Z})=\left\{x=x(k) \in \ell^{\infty}(\mathbb{Z}): x(k) \geq 0\right\} .
$$

As described above, particles with zero mass will be deleted from the system during the evolution. However, relabeling the particle indices whenever a particle vanishes can be problematic. On the one hand, relabeling can be ambiguous, for example the vanishing times might not be in order or could have accumulation points. On the other hand the solution will be discontinuous in time. Thus it is more convenient to leave the configuration unchanged and update the interaction term on the right-hand side of equation (1.1) instead. For this purpose we define the the nearest living neighbour indices

$$
\begin{aligned}
& \sigma_{+}(x, k)=\inf \{l>k: x(l)>0\} \\
& \sigma_{-}(x, k)=\sup \{l<k: x(l)>0\}
\end{aligned}
$$

where we just write $\sigma_{ \pm}(k)$ if there is no danger of confusion. Also we define the ordinary discrete Laplacian $\Delta$ and the living particles Laplacian $\Delta_{\sigma}$ as

$$
\begin{aligned}
\Delta x & =x(k-1)-2 x(k)+x(k), \\
\Delta_{\sigma} x(k) & =\left(x\left(\sigma_{-}(k)\right)-2 x(k)+x\left(\sigma_{+}(k)\right)\right) \cdot \chi_{\{x(k) \neq 0\}} .
\end{aligned}
$$

Then the evolution of the system is governed by the following equation:

$$
\left\{\begin{array}{l}
\partial_{t} x=\Delta_{\sigma} F_{\beta}(x) \quad \text { in }(0, \infty) \times \mathbb{Z} \\
x(0, \cdot)=x_{0}
\end{array}\right.
$$

with $x_{0} \in \ell_{+}^{\infty}$ and

$$
F_{\beta}(x)=-\frac{\beta}{|\beta|} x^{\beta}
$$

with $F_{\beta}(0):=0$ for $\beta<0$. The only drawback is that the right-hand side of $(2.6)$ is no longer continuous, hence we have to use a concept of mild solutions, as in [HNV16]: 


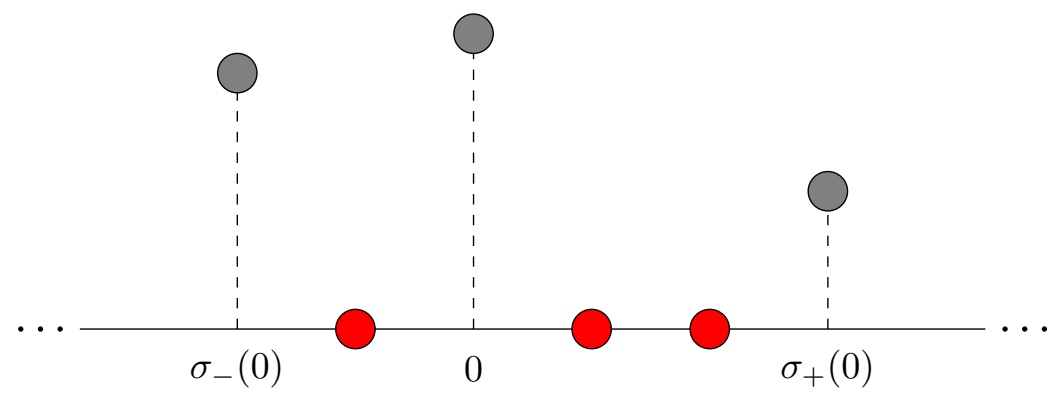

Figure 3: Vanished particles remain in the physical domain, only neighbour relations $\sigma_{+}, \sigma_{-}$change.

Definition 2.1. Let $0<T \leq \infty$. We say that $x:[0, T) \rightarrow \ell_{+}^{\infty}(\mathbb{Z})$ is a solution to problem (2.6) if the following conditions are satisfied:

1. $t \mapsto x(t, k)$ is continuous on $[0, T)$ and $x(0, k)=x_{0}(k)$ for every $k \in \mathbb{Z}$.

2. $t \mapsto F_{\beta}(x) \cdot \chi_{\{x(k) \neq 0\}}$ is locally integrable on $[0, T)$ for every $k \in \mathbb{Z}$.

3. For every $0 \leq t_{1}<t_{2}<T$ and every $k \in \mathbb{Z}$ we have

$$
x\left(t_{1}, k\right)-x\left(t_{2}, k\right)=\int_{t_{1}}^{t_{2}} \Delta_{\sigma} F_{\beta}(x)(s, k) \mathrm{d} s .
$$

The second condition is automatically satisfied if $\beta$ is positive. For the existence of solutions we refer to [HNV16], where the case $\beta<0$ is discussed. We expect a similar result to hold for positive $\beta$ but since we are only concerned with special solutions anyway we will give no proof here. More important for our result is the well-posedness of the time-reversed evolution

$$
\partial_{t} u=\Delta G_{\beta}(u),
$$

with $G_{\beta}(u)=-F_{\beta}(u)$, which is the discrete analogue of a fast diffusion equation. This is addressed in the appendix, see Theorem A.3.

It is easy to check that the evolution (2.6) conserves the average mass

$$
\langle x\rangle=\lim _{N \rightarrow \infty} \frac{1}{2 N+1} \sum_{k=-N}^{N} x(k) .
$$


This is not really meaningful, since vanished and living particles are treated the same. To adequately measure the coarsening process, one has to average only over the living particles. Consequently we define

$$
\begin{aligned}
& L_{\sigma_{+}, N}=\bigcup_{k=1}^{N}\left\{\sigma_{+}^{k}(0)\right\}, \\
& L_{\sigma_{-}, N}=\bigcup_{k=1}^{N}\left\{\sigma_{-}^{k}(0)\right\},
\end{aligned}
$$

as sets of the first $N$ positive, respectively negative living particle particle indices and set

$$
L_{\sigma, N}=\left\{\begin{array}{l}
L_{\sigma_{+}, N} \cup L_{\sigma_{-}, N}, \text { if } x(0)=0, \\
L_{\sigma_{+}, N} \cup L_{\sigma_{-}, N} \cup 0, \text { if } x(0)>0 .
\end{array}\right.
$$

Then we can define the living particle means

$$
\begin{aligned}
\langle x\rangle_{\sigma, N} & =\frac{1}{\left|L_{\sigma, N}\right|} \sum_{k \in L_{\sigma, N}} x(k), \\
\langle x\rangle_{\sigma}^{+} & =\limsup _{N \rightarrow \infty}\langle x\rangle_{\sigma, N}, \\
\langle x\rangle_{\sigma}^{-} & =\liminf _{N \rightarrow \infty}\langle x\rangle_{\sigma, N} .
\end{aligned}
$$

Since mass is transferred from small to large particles and the small particles eventually vanish, we expect the living particle means to grow in time.

\subsection{Main result}

In the main result of the paper we show that there exist solutions where the average particle size grows with the characteristic rate that is indicated by scaling:

Theorem 2.2. Let $\beta \in(-\infty, 0) \cup(0,1]$ and $F_{\beta}$ be defined as above. Then the following statements hold:

1. For every $\beta \in(-\infty, 0) \cup(0,1)$ there exists $x_{0} \in \ell_{+}^{\infty}(\mathbb{Z})$ and a solution to equation (2.6) with initial data $x_{0}$ that satisfies

$$
\begin{aligned}
\langle x\rangle_{\sigma}^{-} & \gtrsim t^{\frac{1}{1-\beta}}, \\
\|x\|_{\infty} & \lesssim t^{\frac{1}{1-\beta}} .
\end{aligned}
$$


2. For $\beta=1$ there exists $0<\lambda \leq 2, x_{0} \in \ell_{+}^{\infty}(\mathbb{Z})$ and a solution to equation (2.6) with initial data $x_{0}$ that satisfies

$$
\begin{aligned}
\langle x\rangle_{\sigma}^{-} & \gtrsim \exp (\lambda t), \\
\|x\|_{\infty} & \lesssim \exp (\lambda t) .
\end{aligned}
$$

Here, $\gtrsim$ and $\lesssim$ mean that the corresponding inequalities hold up to a multiplicative constant that depends only on $\beta$.

In the following we give a short outline of the proof. The key observation is that the time-reversed system corresponding to equation (2.6) is a non-linear parabolic equation where particles are inserted instead of vanishing, which is much easier to handle. Thus the idea is to make a more or less explicit construction in the time-reversed setting and then reverse time again to obtain a sequence of approximate solutions $x^{(n)}$ which solve (2.6) and eventually converge to a solution with the desired properties. Each solution $x^{(n)}$ will be constructed in $n$ steps, starting in the future time $T_{n}$ (with $T_{n} \rightarrow \infty$ ), where the particle sizes are of order $\theta^{n}$ for some $\theta>1$. We then insert particles to lower the average particle size to order $\theta^{n-1}$ and run the time-reversed evolution, equilibrating the system until all particle sizes are of order $\theta^{n-1}$. The procedure is then iterated, going from sizes of order $\theta^{n+1-j}$ to $\theta^{n-j}$, until after $n$ steps all particles sizes are of order one. A suitable compactness argument for $n \rightarrow \infty$ then yields a solution $x$ on $[0, \infty)$ to equation (2.6).

In order to achieve the desired coarsening rate the time-span to equilibrate in the $j$ th step has to be of order $\theta^{(1-\beta)(n+1-j)}$, which is a-priori not clear. Due to scaling however, every step is equivalent to the problem of inserting particles into a configuration $u_{0}$ of order one (denoted by $u_{0} \mapsto \Psi_{*} u_{0}$ ) such that after evolving the system under the backward equation for a uniform timespan $T$ the particles are of order $\theta^{-1}$. More precisely, we will prove the following result, which is the heart of the argument:

Lemma 2.3 (Key Lemma). Let $\beta \in(-\infty, 0) \cup(0,1]$ and $G_{\beta}=-F_{\beta}$. Then for every $\varepsilon>0$ there exists $T=T(\beta, \varepsilon)>0$, such that the following holds: For every $u_{0} \in \ell_{+}^{\infty}(\mathbb{Z})$ with $\frac{1}{2} \leq u_{0} \leq 1$ there exists a creation operator $\Psi_{*}$ and a solution $u$ of the equation

$$
\left\{\begin{array}{l}
\partial_{t} u=\Delta G_{\beta}(u) \quad \text { in }(0, \infty) \times \mathbb{Z}, \\
u(0, \cdot)=\Psi_{*} u_{0}
\end{array}\right.
$$

that satisfies

$$
\left|u(T, .)-\frac{1}{2}\right| \leq \varepsilon
$$




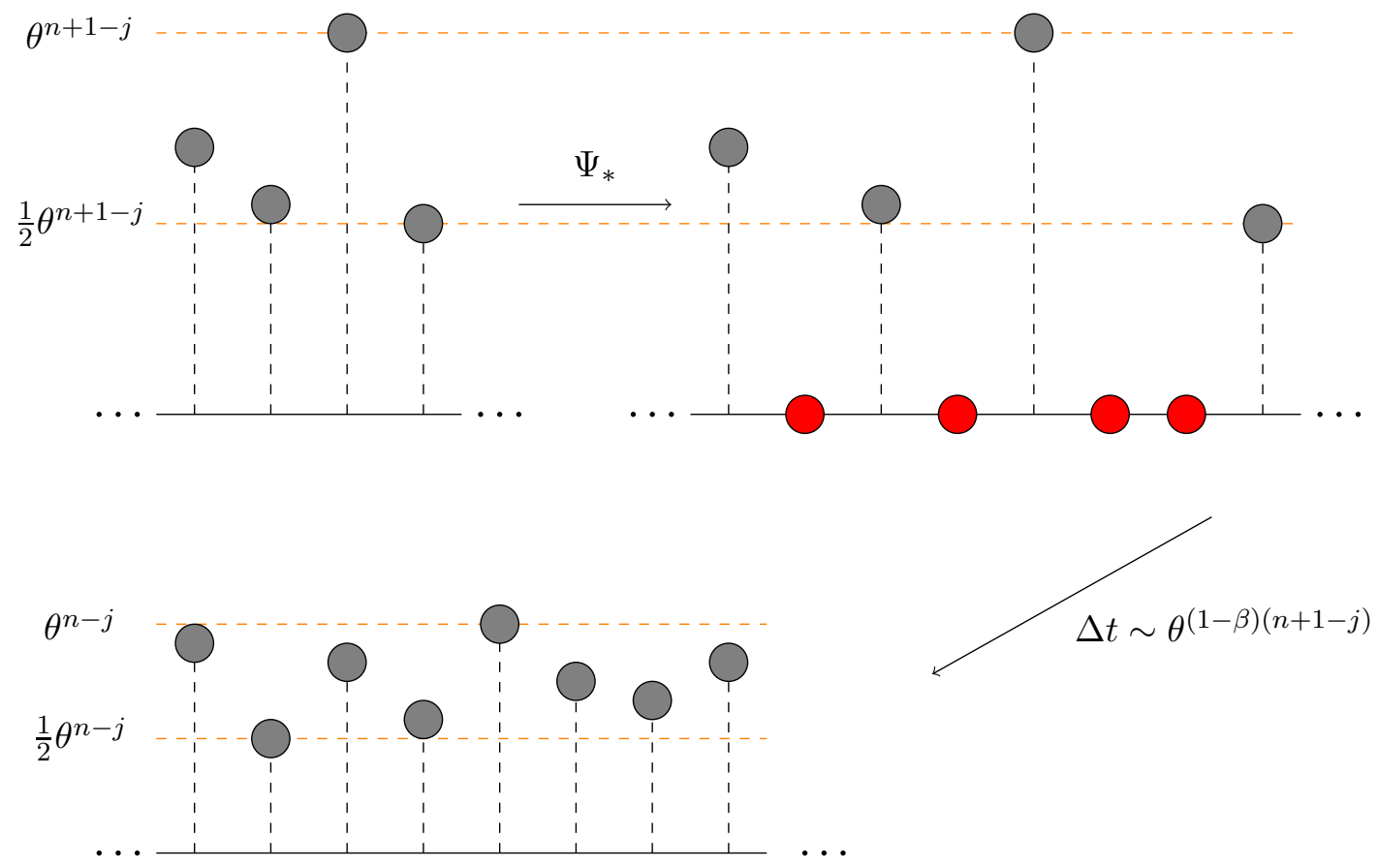

Figure 4: The $j$-th step in the back-in-time construction.

The precise meaning of $\Psi_{*} u_{0}$ will be explained in the next section. In particular, if we set $\theta^{-1}=1 / 2+\varepsilon$ with $\varepsilon \leq 1 / 6$ then $u$ will satisfy the desired estimate

$$
\frac{1}{2} \theta^{-1} \leq u(T, .) \leq \theta^{-1} .
$$

The main idea to prove the lemma is to insert particles such that $1 / 2-\varepsilon \leq \Psi_{*} u_{0} \leq 1 / 2+\varepsilon$ holds in an averaged sense. Since the backward equation is a diffusion, it is expected that the system equilibrates and average-wise estimates eventually induce point-wise estimates after a certain timespan, see Lemma 4.5. Note that due to the freedom of choice in the parameter $\varepsilon$, the back-in-time construction can generate initial data that are arbitrarily flat, demonstrating the instability of constant data:

Corollary 2.4. Let $c>0$. Then for every $\varepsilon>0$ there exist initial data $x_{0}$ as in Theorem 2.2 such that

$$
\left\|x_{0}-c\right\|_{\infty} \leq \varepsilon .
$$

Before proving these results we introduce the formalism $\Psi_{*}$ for the insertion of particles and explain the general construction of solutions to the coarsening equation. 


\section{Construction of solutions}

The general idea to construct (local-in-time) solutions to equation (2.6) is to choose some terminal data $x(T, \cdot)$ and go backward in time from there. The crucial observation is that the vanishing of particles corresponds to the creation of particles if time is reversed. Additionally, since the living particles do not carry any information of the vanished particles in the forward-in-time equation, new particles can be created at arbitrary times $\tau_{j}$ and positions $\left\{\Psi_{*}^{(j)}\right\}$ (for notation see below) in the backward equation. This gives the necessary freedom to construct solutions with desirable properties. Summarizing the above considerations, each data triple $\left(x(T, \cdot),\left\{\tau_{j}\right\},\left\{\Psi_{*}^{(j)}\right\}\right)$ gives rise to a solution of equation (2.6) on the interval $[0, T]$. In the following section we carry this out in detail.

\subsection{Insertion of particles}

First we fix the notation for the insertion of particles. Basically, we need a precise way to insert zeroes into a given sequence of numbers. The most practical way to do this is via push-forward of a suitable increasing map $\Psi: \mathbb{Z} \rightarrow \mathbb{Z}$. This map can be defined by the corresponding sequence of "jumps". We make the following definition:

Definition 3.1. Let $d: \mathbb{Z} \rightarrow \mathbb{N}_{0}$ be a given sequence of jumps. Then define the corresponding deformation as

$$
\begin{aligned}
& \Psi: \mathbb{Z} \rightarrow \mathbb{Z} \\
& \Psi(k)=k+\sum_{m=0}^{k} d(m) .
\end{aligned}
$$

Now for every $x \in \ell_{+}^{\infty}(\mathbb{Z})$ we define the push-forward sequence $\Psi_{*} x$ as

$$
\Psi_{*} x(\Psi(k))=x(k),
$$

for all $k \in \mathbb{Z}$ and $\Psi_{*} x(l)=0$ if $l \notin \operatorname{Im}(\Psi)$.

With this definition, if $x$ represents a particle configuration, then $\Psi_{*} x$ represents the same configuration with new mass-zero particles created. To be more precise, the condition $d(k)=l$ exactly means that we are inserting $l$ new particles between the $k$-th and the $(k \pm$ 1 )-th particle, (depending on the sign of $k$ ). We will refer to the mapping $\Psi_{*}$ as particle creation operator and, to keep notation as compact as possible, not explicitly refer to the deformation $\Psi$ or the specific jump sequence $d$ any more, but rather just state where particles are inserted. This is potentially ambiguous, for instance, "creating a particle between each two living particles" can be achieved by different $d$, potentially translating the original living particles. However, in the following sections these ambiguities do not affect the arguments, hence we will ignore them. 


\subsection{Back-in-time construction}

Next we describe how to obtain a solution from a given terminal configuration $x_{\text {ter }}$, an increasing sequence of vanishing/creation times $\left\{\tau_{j}\right\}_{j=1, . ., n}$ and corresponding creation operators $\left\{\Psi_{*}^{(j)}\right\}_{j=1, . ., n}$. We define the solution piecewise by iteratively using the backward evolution (2.22) on $\left[\tau_{j-1}, \tau_{j}\right]$ after inserting particles at $t=\tau_{j-1}$ and continuing the procedure. To be precise, we define $u^{(j)}$ on the interval $\left[\tau_{j-1}, \tau_{j}\right]$ to be a solution of the following problem:

$$
\left\{\begin{array}{l}
\partial_{t} u^{(j)}=\Delta G_{\beta}\left(u^{(j)}\right) \text { in }\left(\tau_{j-1}, \tau_{j}\right] \times \mathbb{Z}, \\
u^{(j)}\left(\tau_{j-1}\right)=\Psi_{*}^{(j)}\left[u^{(j-1)}\left(\tau_{j-1}\right)\right]
\end{array}\right.
$$

for $j=1, . ., n$, with $\tau_{0}:=0, u^{(0)}\left(\tau_{0}\right):=x_{\text {ter }}$ and $G_{\beta}=-F_{\beta}$. We should note that by a solution we mean a classical solution, i.e $u^{(j)} \in C^{0}\left(\left[\tau_{j-1}, \infty\right), \ell_{+}^{\infty}(\mathbb{Z})\right)$, for every $k \in \mathbb{Z}$ we have $u^{(j)}(., k) \in C^{1}\left(\left(\tau_{j-1}, \infty\right)\right)$ and the equation holds pointwise. Well-posedness of this problem is a-priori not clear, especially for the case $\beta<0$. For the moment we just assume that the equation is solvable and focus on carrying out the construction of solutions to the coarsening equation. In Theorem A.3 we give a sufficient condition on the initial data for existence of solutions that is easily verified for the data considered in the next section.

Reversing the time direction we obtain piecewise solutions of our original equation. However, one has to compose $u^{(j)}$ with the creation operators once more, since vanished particles remain in the "physical" domain in the original evolution (2.6). To be more precise, we set

$$
x^{(j)}(t)=\left(\prod_{l=1}^{j-1} \Psi_{*}^{(n+1-l)}\right)\left[u^{(n+1-j)}\left(\tau_{n}-t\right)\right],
$$

which lets us glue the solutions together in a continuous way:

$$
x(t)=x^{(j)}(t), \text { if } t \in\left[\tau_{n}-\tau_{n+1-j}, \tau_{n}-\tau_{n-j}\right),
$$

for $j=1, . ., n$. Using $u^{(j)}\left(\tau_{j-1}\right)=\Psi_{*}^{(j)}\left[u^{(j-1)}\left(\tau_{j-1}\right)\right]$ it is easy to check that $x$ defined this way is continuous in time. The next lemma shows that $x$ is indeed a solution to our original equation:

Lemma 3.2. Let $\Psi_{*}$ be a creation operator as above. Then we have

$$
\text { 1. } \sigma_{ \pm}\left(\Psi_{*} x, \Psi(k)\right)=\Psi\left(\sigma_{ \pm}(x, k)\right) \text { for every } x \in \ell_{+}^{\infty} \text { and } k \in \mathbb{Z} \text {. }
$$


2. $\left[\Delta_{\sigma}, \Psi_{*}\right] x=\left(\Delta_{\sigma} \Psi_{*}-\Psi_{*} \Delta_{\sigma}\right) x=0$ for every $x \in \ell_{+}^{\infty}$.

3. $\left\langle\Psi_{*} x\right\rangle_{\sigma, N}=\langle x\rangle_{\sigma, N}$ for every $N>0$ and $x \in \ell_{+}^{\infty}$.

Proof. 1. It suffices to prove the claim for $\sigma_{+}$, the other case is completely analogous. Because $\Psi$ is strictly increasing, we have $\Psi\left(\sigma_{+}(x, k)\right)>\Psi(k)$. We also have

$$
\Psi_{*} x\left(\Psi\left(\sigma_{+}(x, k)\right)\right)=x\left(\sigma_{+}(x, k)\right)>0,
$$

which shows $\sigma_{+}\left(\Psi_{*} x, \Psi(k)\right) \leq \Psi\left(\sigma_{+}(x, k)\right)$. For the other inequality, we note that $\Psi_{*} x(l)>0$ implies that $l=\Psi(m)$ for some $m \in \mathbb{Z}$. In this case we have

$$
0<\Psi_{*} x(l)=x(m)
$$

which implies $m \geq \sigma_{+}(x, k)$, and because $\Psi$ is increasing we conclude

$$
l=\Psi(m) \geq \Psi\left(\sigma_{+}(x, k)\right),
$$

which proves the first assertion.

2. Let $l=\Psi(k)$. We apply the identity in 1 . to get

$$
\begin{aligned}
\Delta_{\sigma} \Psi_{*} x(l) & =\left(\Psi_{*} x\left(\sigma_{-}\left(\Psi_{*} x, l\right)\right)-2 \Psi_{*} x(l)+\Psi_{*} x\left(\sigma_{+}\left(\Psi_{*} x, l\right)\right)\right) \cdot \chi_{\left\{\Psi_{*} x(l) \neq 0\right\}} \\
& =\left(x\left(\sigma_{-}(k)\right)-2 x(k)+x\left(\sigma_{+}(k)\right)\right) \cdot \chi_{\{x(k) \neq 0\}} \\
& =\Delta_{\sigma} x(k)=\Psi_{*} \Delta_{\sigma} x(l) .
\end{aligned}
$$

On the other hand, if $l \notin \operatorname{Im}(\Psi)$, the identity is trivial.

3. Obvious from the definition.

With the second statement of the above lemma, it is not difficult to verify that the sequence $x$ we have constructed above solves equation (2.6):

Corollary 3.3. Let $x_{\mathrm{ter}},\left\{\tau_{j}\right\}$ and $\left\{\Psi_{*}^{(j)}\right\}$ be given and $x$ be constructed as above. If $t \mapsto F_{\beta}(x) \cdot \chi_{\{x(k) \neq 0\}}$ is locally integrable for every $k \in \mathbb{Z}$, then $x$ is a (mild) solution to equation (2.6) on $\left[0, \tau_{n}\right)$.

Proof. Since $x$ is continuous and piecewise smooth by construction, it suffices to show that $\partial_{t} x=\Delta_{\sigma} F_{\beta}(x)$ holds pointwise on all intervals $\left[\tau_{n}-\tau_{n+1-j}, \tau_{n}-\tau_{n-j}\right)$. Indeed, we calculate 


$$
\begin{aligned}
\partial_{t} x^{(j)}(t) & =\left(\prod_{l=1}^{j-1} \Psi_{*}^{(n+1-l)}\right)\left[\partial_{t} u^{(n+1-j)}\left(\tau_{n}-t\right)\right] \\
& =\left(\prod_{l=1}^{j-1} \Psi_{*}^{(n+1-l)}\right)\left[\Delta_{\sigma} F_{\beta}\left(u^{(n+1-j)}\right)\left(\tau_{n}-t\right)\right] \\
& =\Delta_{\sigma} F_{\beta}\left(\left(\prod_{l=1}^{j-1} \Psi_{*}^{(n+1-l)}\right)\left[u^{(n+1-j)}\left(\tau_{n}-t\right)\right]\right)=\Delta_{\sigma} F_{\beta}\left(x^{(j)}\right) .
\end{aligned}
$$

Here we used that $\Delta_{\sigma}$ commutes with creation operators by the previous lemma, as well as composition with the function $F_{\beta}$.

Remark. The above construction scheme implies the existence of many initial data and corresponding solutions to the coarsening equation which become stationary after a finite time. Indeed, $x$ as above has this property if we pick $x_{\text {ter }}$ to be a constant sequence. Because there is much freedom in the choice of particle creations and vanishing times this means that finding conditions on initial data such that lower coarsening bounds hold is a difficult task and remains an open problem. In the construction for the proof of Theorem 2.2 we will in fact choose $x_{\text {ter }}(k)=\theta^{n}$ so that each approximate solution becomes stationary. Because $\theta^{n} \rightarrow \infty$ and $\tau_{n} \rightarrow \infty$ the limit solution however will grow indefinitely. The details will be explained in the next section.

\section{Proof of the main result}

We divide the full proof of Theorem 2.2 into four main steps. In the first step we show how to insert particles to modify the local average in a uniform way. The second step is to prove a long-time diffusive property of the backward equation which, together with the first step, will enable us to prove Lemma 2.3. In the third step the construction of the approximate sequence $x^{(n)}$ is thoroughly carried out. Finally we use a compactness argument to pass to the limit and obtain a solution with the desired properties, finishing the proof.

\subsection{Step 1: Average modification by particle insertion}

Definition 4.1 (Local Averages). Let $x \in \ell^{\infty}(\mathbb{Z})$. Then define the associated sequence of local averages as

$$
\Lambda(x, k, N)=\frac{1}{2 N+1} \sum_{l=-N}^{N} x(k-l) .
$$

In the next lemma we show how to modify the local averages of a given sequence by inserting particles in a suitable way: 
Lemma 4.2 (Particle insertion). Let $u_{0} \in \ell_{+}^{\infty}(\mathbb{Z})$ with

$$
\frac{1}{2} \leq u_{0} \leq 1
$$

Then for every $\varepsilon>0$ there exists a creation operator $\Psi_{*}$ and $N_{0} \in \mathbb{N}$ such that

$$
\left|\Lambda\left(\Psi_{*} u_{0}, ., N\right)-\frac{1}{2}\right| \leq \varepsilon
$$

for $N \geq N_{0}$. Furthermore, if $d$ is the jump sequence associated to $\Psi_{*}$, then $\|d\|_{\infty}$ is finite and depends only on $\varepsilon$.

Proof. Let $\left(\lambda_{i}\right)$ be an equidistant partition of the interval $[1 / 2,1]$ with $\left|\lambda_{i}-\lambda_{i+1}\right| \leq \varepsilon$. We give an explicit scheme for the particle insertion as follows: We divide $\mathbb{Z}$ into disjoint blocks of particles with length $K$, where $K$ is determined later:

$$
\mathbb{Z}=\bigcup_{j \in \mathbb{Z}} B_{j}
$$

with $B_{j}=\{j K, \ldots,(j+1) K-1\}$. Let $\Lambda_{j}$ denote the average mass in $B_{j}$ with respect to $u_{0}$. We define the deformation $\Psi$ by inserting $L_{i}$ (determined later) particles to the right of $(j+1) K-1$ whenever he have

$$
\lambda_{i} \leq \Lambda_{j} \leq \lambda_{i+1}
$$

This gives rise to a new partition of $\mathbb{Z}$ into blocks $\tilde{B}_{j}$ with varying lenghts $K+L_{i}$, where $\tilde{B}_{j}$ contains all elements of $\Psi\left(B_{j}\right)$ and the next $L_{i}$ numbers that are not elements of $\operatorname{Im}(\Psi)$. We call a block with $L_{i}$ inserted particles a block of the $i$-th kind. Then the average mass $\tilde{\Lambda}_{j}$ of such a block with respect to $\Psi_{*} u_{0}$ is by construction

$$
\tilde{\Lambda}_{j}=\frac{1}{K+L_{i}}\left(\sum_{k \in B_{j}} u_{0}(k)\right)=\frac{K}{K+L_{i}} \Lambda_{j}
$$

which gives

$$
\lambda_{i} \frac{K}{K+L_{i}} \leq \tilde{\Lambda}_{j} \leq \lambda_{i+1} \frac{K}{K+L_{i}}:=\lambda_{i+1} \theta_{i} .
$$

Because $1 / 2 \leq \lambda_{i} \leq 1$ we can, if $K$ is large enough, choose $L_{i} \leq K$ such that 


$$
\left|\lambda_{i} \theta_{i}-\frac{1}{2}\right| \leq \mathcal{O}(\varepsilon)
$$

and because $\lambda_{i}$ and $\lambda_{i+1}$ are close we also have

$$
\left|\lambda_{i+1} \theta_{i}-\frac{1}{2}\right| \leq \mathcal{O}(\varepsilon)
$$

This implies that the average mass of every block $\tilde{B}_{j}$ can be estimated as

$$
\left|\tilde{\Lambda}_{j}-\frac{1}{2}\right| \leq \mathcal{O}(\varepsilon)
$$

Next we calculate $\Lambda\left(\Psi_{*} u_{0}, k, N\right)$ for $N \gg K$ and arbitrary $k \in \mathbb{Z}$. Denote by $n_{i}$ the number of blocks of the $i$-th kind in the domain of summation, that is $\{k-N, \ldots, k+N\}$. This implies that

$$
|\{k-N, \ldots, k+N\}|=2 N+1=\sum_{i}\left(K+L_{i}\right) n_{i}+\mathcal{O}(K) .
$$

Then we divide the summation in $\Lambda\left(\Psi_{*} u_{0}, k, N\right)$ into summation over the respective blocks and the rest of the particles in $\{k-N, \ldots, k+N\}$, whose number, and thus total mass $R$, is of order $K$. Thus we have

$$
\begin{aligned}
\Lambda\left(\Psi_{*} u_{0}, k, N\right) & =\frac{1}{2 N+1}\left(\sum_{\text {sum over blocks }}+R\right) \\
& =(1+\mathcal{O}(K / N)) \frac{\sum_{\text {sum over blocks }}}{\sum_{i}\left(K+L_{i}\right) n_{i}}+\mathcal{O}(K / N) .
\end{aligned}
$$

By the estimates on the average masses of the blocks we have

$$
\left(\frac{1}{2}-\varepsilon\right) \sum_{i}\left(K+L_{i}\right) n_{i} \leq \sum_{\text {sum over blocks }} \leq\left(\frac{1}{2}+\varepsilon\right) \sum_{i}\left(K+L_{i}\right) n_{i},
$$

which implies the desired estimate if $K / N \leq \mathcal{O}(\varepsilon)$. Because $L_{i} \leq K$ by construction the jump sequence satisfies $d \leq K$ and $K$ depends only on $\varepsilon$. 


\subsection{Step 2: Estimate for the backward equation}

The basic idea to analyse equation (2.22) is to view it as a discrete parabolic equation in divergence form with time-dependent coefficients. More precisely, with the finite difference operators

$$
\begin{aligned}
& \partial^{+} u(k)=u(k+1)-u(k), \\
& \partial^{-} u(k)=u(k)-u(k-1),
\end{aligned}
$$

we calculate

$$
\partial_{t} u=\Delta G_{\beta}(u)=\partial^{-} \partial^{+}\left(G_{\beta}(u)\right)=\partial^{-}\left(a \partial^{+} u\right),
$$

where

$$
a(t, k)=a_{\beta}(t, k)=\frac{G_{\beta}(u(t, k+1))-G_{\beta}(u(t, k))}{u(t, k+1)-u(t, k)} .
$$

The coefficient $a$ is strictly positive and bounded from below if $u$ is bounded from above but becomes singular at $u=0$, except for $\beta=1$, where $a=1$. Because of this we want to work with solutions that are bounded from above and below:

Lemma 4.3. (Positivity estimate) Let $\beta \in(-\infty, 0) \cup(0,1)$ and $u_{0} \in \ell_{+}^{\infty}(\mathbb{Z})$ with $\frac{1}{2} \leq$ $u_{0} \leq 1$. Let $\Psi_{*}$ be a creation operator with associated jump sequence $d$ that satisfies $d(k) \leq L$. Then there exists a (classical) solution to equation (2.22) with initial data $\Psi_{*} u_{0}$. Furthermore, we have $u \leq 1$ and

$$
u(t, \cdot) \geq c\left(1 \wedge t^{\frac{1}{1-\beta}}\right),
$$

where $c$ depends only on $\beta$ and $L$.

Proof. Because the jump sequence satisfies $d \leq L$, the distance between particles that have mass at least $1 / 2$ is at most $L+1$. Then the result follows directly from Theorem A.3. since the above considerations imply $\Psi_{*} u_{0} \in \mathcal{P}_{L+1, \frac{1}{2}}$.

The lemma implies that there exists a solution $u$ such that equation (2.22) is immediately strictly parabolic. Before we turn to the analysis of linear parabolic equations we establish uniform Hölder continuity. This is important for the stability of local averages for small times and later for the compactness of the approximating sequence.

Lemma 4.4. (Uniform Hölder continuity) Let $u_{0}, \beta, \Psi_{*}$ and $u$ be as above. Then the following statements hold: 
1. For $\beta<0$ and $T>0$ there exists $C=C(\beta, L, T)$ such that

$$
\left|u\left(t_{2}, k\right)-u\left(t_{1}, k\right)\right| \leq C\left|t_{2}-t_{1}\right|^{\frac{1}{1-\beta}}
$$

for all $0<t \leq T$ and $k \in \mathbb{Z}$.

2. For $\beta \in(0,1]$ we have

$$
\left|u\left(t_{2}, k\right)-u\left(t_{1}, k\right)\right| \leq 4\left|t_{2}-t_{1}\right|
$$

for all $t>0$ and $k \in \mathbb{Z}$.

Proof. First we note that due to equation (2.22) we have for $t_{2}>t_{1}$ :

$$
\left|u\left(t_{2}, k\right)-u\left(t_{1}, k\right)\right| \leq \int_{t_{1}}^{t_{2}}\left|\Delta G_{\beta}(u)(s, k)\right| \mathrm{d} s .
$$

The estimate $u \leq 1$ implies $\left|\Delta G_{\beta}(u)\right| \leq 4$ if $\beta \in(0,1]$. For negative $\beta$ we use the lower bound (4.19) to get $\left|\Delta G_{\beta}(u)(s, k)\right| \leq C(\beta, L, T) s^{\frac{\beta}{1-\beta}}$ on each compact interval $[0, T]$. Then the desired inequality follows by using the estimates on $\Delta G_{\beta}(u)$ in the above identity and the elementary inequality $a^{\frac{1}{1-\beta}}-b^{\frac{1}{1-\beta}} \leq(a-b)^{\frac{1}{1-\beta}}$ for $a \geq b$ and $\beta<0$.

The next step is the long-time diffusivity result for linear equations, making use of discrete parabolic Hölder regularity (see appendix for details).

Lemma 4.5. (Long-time estimate) Let $a:[0, \infty) \rightarrow \ell_{+}^{\infty}(\mathbb{Z})$ with $0<\lambda_{1} \leq a \leq \lambda_{2}$ and $a(., k) \in C^{0}([0, \infty))$ for every $k \in \mathbb{Z}$. Let $u_{0} \in \ell_{+}^{\infty}(\mathbb{Z})$ and assume that there exist positive constants $c_{1}, c_{2}$ such that $c_{1} \leq \Lambda\left(u_{0}, ., N\right) \leq c_{2}$ for $N \geq N_{0}$. Let $u$ be the solution of

$$
\left\{\begin{array}{l}
\partial_{t} u=\partial^{-}\left(a \partial^{+} u\right)=\mathcal{L}(t) u \\
u(0, .)=u_{0} .
\end{array}\right.
$$

Then for every $\varepsilon>0$ there exists $T=T(\varepsilon)>0$, depending only on $N_{0}$ and the bounds on a, such that

$$
c_{1}-\varepsilon \leq u(t, k) \leq c_{2}+\varepsilon
$$

for all $t \geq T, k \in \mathbb{Z}$. 
Proof. Since spatial translation does not change the type of equation and the bounds on $a$, it suffices to estimate $u(t, 0)$. Let $\phi=\phi(t, k, s, l)$ denote the full fundamental solution to equation (4.22). Then we have

$$
u(t, 0)=\sum_{l} \phi(t, 0,0, l) u_{0}(l)=\int_{\mathbb{R}} U(t, \xi) u_{0}\left(\left\lfloor t^{\frac{1}{2}} \xi\right\rfloor\right) \mathrm{d} \xi
$$

with

$$
U(t, \xi)=t^{\frac{1}{2}} \phi\left(t, 0,0,\left\lfloor t^{\frac{1}{2}} \xi\right\rfloor\right) .
$$

Let $\varepsilon>0$. By Corollary A.19 there exist $T, \delta>0$ depending only on $\varepsilon$ and the bounds on $a$ such that $U(t,$.$) can be approximated in L^{1}$ by step-functions (which can be chosen to be positive since $\phi$ is positive) of step-width $\delta$ up to an error of $\varepsilon$ for $t \geq T$. Let $\chi=\sum_{k} a_{k} \chi_{I_{k}}$ be such a step-function, then we calculate

$$
\begin{aligned}
\int_{\mathbb{R}} \chi(\xi) u_{0}\left(\left\lfloor t^{\frac{1}{2}} \xi\right\rfloor\right) \mathrm{d} \xi & =\sum_{k} a_{k} \int_{I_{k}} u_{0}\left(\left\lfloor t^{\frac{1}{2}} \xi\right\rfloor\right) \mathrm{d} \xi \\
& =\sum_{k}\left|I_{k}\right| a_{k} \frac{1}{t^{\frac{1}{2}}\left|I_{k}\right|} \int_{t^{\frac{1}{2}} I_{k}} u_{0}(\lfloor\xi\rfloor) \mathrm{d} \xi
\end{aligned}
$$

Since $c_{1} \leq \Lambda\left(u_{0}, ., N\right) \leq c_{2}$ for $N \geq N_{0}$, we have

$$
c_{1}-\varepsilon \leq \frac{1}{2 R} \int_{[-R, R]} u_{0}(\lfloor\xi-\eta\rfloor) \mathrm{d} \eta \leq c_{2}+\varepsilon,
$$

for large enough $R$. Hence we have

$$
\left(c_{1}-\varepsilon\right)\|\chi\|_{L^{1}} \leq \int_{\mathbb{R}} \chi(\xi) u_{0}\left(\left\lfloor t^{\frac{1}{2}} \xi\right\rfloor\right) \mathrm{d} \xi \leq\left(c_{2}+\varepsilon\right)\|\chi\|_{L^{1}},
$$

for large enough $t \geq T$, since $\left|I_{k}\right| \geq \delta$. For such $t$ and $\chi$ approximating $U$ we calculate

$$
\begin{aligned}
\int_{\mathbb{R}} U(t, \xi) u_{0}\left(\left\lfloor t^{\frac{1}{2}} \xi\right\rfloor\right) \mathrm{d} \xi & \geq\left(c_{1}-\varepsilon\right)\|\chi\|_{L^{1}}-\left\|u_{0}\right\|_{\infty}\|U(t, .)-\chi\|_{L^{1}} \\
& =c_{1}+\mathcal{O}(\varepsilon),
\end{aligned}
$$

where we used $\|\chi\|_{L^{1}}=\|U(t, .)\|_{L^{1}}+\mathcal{O}(\varepsilon)=1+\mathcal{O}(\varepsilon)$ in the last step. The other bound is analogous.

Combining these two results we can prove the Key Lemma 2.3 , 
Proof of Lemma 2.3. Let $\varepsilon>0$. By Lemma 4.2 there exists $\Psi_{*}$ (with $\|d\|_{\infty}$ depending on $\varepsilon$ ) and $N_{0}=N_{0}(\varepsilon)$ such that

$$
\frac{1}{2}-\varepsilon \leq \Lambda\left(\Psi_{*} u_{0}, \cdot, N\right) \leq \frac{1}{2}+\varepsilon
$$

for $N \geq N_{0}$. Because of uniform Hölder continuity (see Lemma 4.4) there exists $t_{1}=$ $t_{1}(\beta, \varepsilon)>0$ such that

$$
\frac{1}{2}-2 \varepsilon \leq \Lambda\left(u\left(t_{1}, \cdot\right), \cdot, N\right) \leq \frac{1}{2}+2 \varepsilon
$$

On the other hand, Lemma 4.3 implies that

$$
u\left(t_{1}, \cdot\right) \geq \delta=\delta(\beta, \varepsilon)>0,
$$

for all $t \geq t_{1}$. In particular, equation (2.22) is strictly parabolic on $\left(t_{1}, \infty\right)$ (for $\beta=1$ this step is obsolete). Then according to Lemma 4.5 there exists $t_{2}=t_{2}(\beta, \varepsilon)$ such that

$$
\frac{1}{2}-3 \varepsilon \leq u(T, \cdot) \leq \frac{1}{2}+3 \varepsilon
$$

with $T=t_{2}+t_{1}$.

\subsection{Step 3: Approximating sequence}

We will now construct the approximating sequence $x^{(n)}$, using the technique established in Section 3. Thus for every $n$ we have to specify the terminal data, creation times $\tau_{n, j}$ and creation operators $\Psi_{*}^{(n, j)}$. For the rest of the section we fix $0<\varepsilon \leq 1 / 6$ and define

$$
\theta^{-1}=\frac{1}{2}+\varepsilon
$$

Let $T$ be as in Lemma 2.3 and set

$$
x_{\text {ter }}^{(n)}=\theta^{n},
$$

a constant sequence. Now $\tau_{n, j}$ and $\Psi_{*}^{(n, j)}$ are constructed iteratively. We choose $\Psi_{*}^{(n, 1)}$ according to Lemma 2.3 applied to the constant sequence 1 , then by the statement of the lemma, the fact that $1 / 2-\varepsilon \geq \theta^{-1} / 2$ and the scaling properties of the equation we have 


$$
\frac{1}{2} \theta^{n-1} \leq u^{(n, 1)}\left(T \theta^{(1-\beta) n}, \cdot\right) \leq \theta^{n-1}
$$

where $u^{(n, 1)}$ is a solution to the backward equation (2.22) with initial data $\Psi_{*}^{(n, 1)} x_{\text {ter }}^{(n)}$ according to Lemma 2.3. Consequently we set

$$
\tau_{n, 1}=T \theta^{(1-\beta) n} .
$$

Iterating the procedure, for given $\tau_{n, j-1}$ and $u^{(n, j-1)}$ with

$$
\frac{1}{2} \theta^{n-j+1} \leq u^{(n, j-1)}\left(\tau_{n, j-1}, .\right) \leq \theta^{n-j+1},
$$

we apply Lemma 2.3 to the rescaled sequence

$$
\theta^{-n+j-1} u^{(n, j-1)}\left(\tau_{n, j-1}, .\right)
$$

which yields a creation operator $\Psi_{*}=: \Psi_{*}^{(n, j)}$ and a solution $u^{(n, j)}$ to

$$
\left\{\begin{array}{l}
\partial_{t} u^{(n, j)}=\Delta G_{\beta}\left(u^{(n, j)}\right) \text { in }\left(\tau_{j-1}, \tau_{j}\right] \times \mathbb{Z} \\
u^{(n, j)}\left(\tau_{n, j-1}\right)=\Psi_{*}^{(n, j)}\left[u^{(n, j-1)}\left(\tau_{n, j-1}\right)\right]
\end{array},\right.
$$

that, by scaling, satisfies

$$
\frac{1}{2} \theta^{n-j} \leq u^{(n, j)}\left(\tau_{n, j}, .\right) \leq \theta^{n-j},
$$

with

$$
\tau_{n, j}=\tau_{n, j-1}+T \theta^{(1-\beta)(n+1-j)} .
$$

As described in the previous section, this procedure yields a solution $x^{(n)}$ on the interval $\left[0, \tau_{n, n}\right]$ to the coarsening equation. The local integrability condition for negative $\beta$ is satisfied due to (4.19). Since $x^{(n)}\left(\tau_{n, n}\right)$ is constant up to vanished particles, the solution can be extended to $[0, \infty)$. Let

$$
\begin{aligned}
T_{n} & =\tau_{n, n}, \\
t_{j} & =T_{n}-\tau_{n, n-j}=T \sum_{k=n+1-j}^{n} \theta^{(1-\beta)(n+1-k)}=T \sum_{m=1}^{j} \theta^{(1-\beta) m} .
\end{aligned}
$$


The numbers $t_{j}$ are exactly the times where particles can vanish and are the same for all $n$. In particular, the vanishing times of the limit will be contained in the set $\left\{t_{j}\right\}$. We summarize the properties of of $x^{(n)}$ that follow directly by construction:

$$
\begin{aligned}
& \partial_{t} x^{(n)}=\Delta_{\sigma} F_{\beta}\left(x^{(n)}\right) \text { in }(0, \infty) \times \mathbb{Z}, \\
& x^{(n)}(t, k)=\theta^{n} \text { for all } t \geq T_{n} \text { and } x^{(n)}(t, k)>0, \\
& x^{(n)}(t, k) \leq \theta^{j} \text { for all } t_{j-1} \leq t \leq t_{j} \text { and } k \in \mathbb{Z}, \\
& x^{(n)}\left(t_{j}, k\right) \geq \frac{1}{2} \theta^{j} \text { for all } 1 \leq j \leq n \text { and } k \in \mathbb{Z} \text { with } x^{(n)}\left(t_{j}, k\right)>0 .
\end{aligned}
$$

\subsection{Step 4: Passage to the limit}

Before using an appropriate compactness argument on the approximating sequence it is also necessary to control the decay of particles near their vanishing times uniformly since the particle interaction is discontinuous at $x(k)=0 . \mathrm{y}$

Lemma 4.6. Let $x^{(n)}$ be defined as above and $\beta \neq 1$. For every $j>0$ there exists $C=C(j, \beta)$ and $\varepsilon=\varepsilon(j, \beta)>0$, such that for all particles $k \in \mathbb{Z}$ that vanish at $t=t_{j}$ we have

$$
x^{(n)}(t, k) \geq C\left(t_{j}-t\right)^{\frac{1}{1-\beta}},
$$

for $t \in\left[t_{j}-\varepsilon, t_{j}\right]$. For $\beta=1$ the statement holds in the same way except we have the lower bound

$$
x^{(n)}(t, k) \geq C \exp \left(-2\left(t_{j}-t\right)\right) I_{L}\left(2\left(t_{j}-t\right)\right),
$$

where $I_{L}$ is the $L$-th modified Bessel function of the first kind and $L$ depends only on $\beta$.

Proof. The construction of $x^{(n)}$ and Lemma 4.3 directly imply the statement for $\beta \neq 1$. The inequality for $\beta=1$ follows from Theorem A.3 in the same way as Lemma 4.3.

With the previous preparation we can prove the main result:

Proof of Theorem 2.2. Using the explicit construction of the sequence $x^{(n)}$ and Lemma 4.4 it is easy to see that $x^{(n)}(\cdot, k)$ is uniformly Hölder continuous on $[0, T]$ for every $k \in \mathbb{Z}$ and $T>0$ defined above. By the Arzela-Ascoli Theorem we can, after an exhaustion $T_{N} \nearrow+\infty$ and a diagonal argument, extract a subsequence (not renamed) and a limit $x=x(t, k)$ such that

$$
x^{(n)}(t, k) \rightarrow x(t, k) \text { as } n \rightarrow \infty \text { for all } t \in[0, \infty) \text { and } k \in \mathbb{Z},
$$


and $x(., k) \in C([0, \infty))$ for all $k \in \mathbb{Z}$. Let

$$
\eta_{k}^{(n)}=\inf \left\{t>0: x^{(n)}(t, k)=0\right\}
$$

denote the vanishing time of the $k$-th particle. By another diagonal argument we can further restrict ourselves to a subsequence such that the particle vanishing times $\left\{\eta_{k}^{(n)}\right\}$ converge:

$$
\eta_{k}^{(n)} \rightarrow \eta_{k} \in\left\{t_{j}\right\}_{j=0, . ., \infty} \cup+\infty \text { as } n \rightarrow \infty \text { for all } k \in \mathbb{Z}
$$

In fact if $\eta_{k}^{(n)}$ is bounded we even have $\eta_{k}^{(n)}=\eta_{k}$ for $n$ large enough because the set $\left\{t_{j}\right\}$ is discrete. Otherwise we can assume $\eta_{k}^{(n)} \rightarrow+\infty$ increasingly. If $\eta_{k}=t_{j}$ we check that this is indeed the vanishing time of $x(., k)$ :

$$
0=\lim _{n \rightarrow \infty} x^{(n)}(t, k)=x(t, k),
$$

for every $t>\eta_{k}$. Furthermore, by Lemma 4.6 we have for $\beta \neq 1$

$$
x^{(n)}(t, k) \geq C\left(t_{j}-t\right)^{\frac{1}{1-\beta}}>0 \text { for all } t \in\left[t_{j}-\varepsilon, t_{j}\right),
$$

for large enough $n$, hence also $x(t, k)>0$ for $t<t_{j}$, and the analogous argument works for $\beta=1$ with the corresponding estimate. Next we show that $x$ solves equation (2.6). Fix $k \in \mathbb{Z}, 0 \leq j_{1}<j_{2}$ and integrate equation (2.6) with $x^{(n)}$ from $s_{1}$ to $s_{2}$, where $t_{j_{1}} \leq s_{1}<s_{2} \leq t_{j_{2}}$, to obtain

$$
x^{(n)}\left(s_{2}, k\right)-x^{(n)}\left(s_{1}, k\right)=\int_{s_{1}}^{s_{2}} \Delta_{\sigma} F_{\beta}\left(x^{(n)}\right)(t, k) \mathrm{d} t .
$$

By construction, the function $t \mapsto \sigma_{ \pm}\left(x^{(n)}(t,), k.\right)$ is constant on $\left(s_{1}, s_{2}\right)$. Furthermore, by the construction of the sequence $x^{(n)}$, the number of values of $\sigma_{ \pm}\left(x^{(n)}(t,), k.\right)$ regarded as a sequence in $n$ is finite, hence we can assume this to be independent of $n$ after taking another subsequence, in other words

$$
\sigma_{ \pm}\left(x^{(n)}(t, .), k\right)=\sigma_{ \pm}(x(t, .), k) \text { for all } s_{1}<t<s_{2} .
$$

Let $s_{1}<t<s_{2}$. If $x(t, k)>0$, then also $x^{(n)}(t, k)>0$ for large $n$ and by the point-wise convergence of $x^{(n)}$ and the above identity we conclude

$$
\Delta_{\sigma} F_{\beta}\left(x^{(n)}\right)(t, k) \rightarrow \Delta_{\sigma} F_{\beta}(x(t, k)) \text { as } n \rightarrow \infty .
$$


On the other hand, $x(t, k)=0$ implies $\eta_{k} \leq t_{j_{1}}$ and $x^{(n)}(t, k)=0$, since $\eta_{k}^{(n)}=\eta_{k}$ for $n$ large, hence we also get $\Delta_{\sigma} F_{\beta}\left(x^{(n)}\right)(t, k) \rightarrow \Delta_{\sigma} F_{\beta}(x(t, k))$. Then we can apply dominated convergence, where we use that $x^{(n)}$ is locally bounded in the case $\beta>0$ and the lower bound from Lemma 4.6] in the case $\beta<0$, and pass to the limit in the above integral identity to conclude

$$
x\left(s_{2}, k\right)-x\left(s_{1}, k\right)=\int_{s_{1}}^{s_{2}} \Delta_{\sigma} F_{\beta}(x)(t, k) \mathrm{d} t,
$$

which shows that $x$ is a solution to the coarsening equation.

It remains to show that $x$ satisfies the desired bounds. We first consider the case $\beta \neq 1$. By (4.49) we have

$$
x^{(n)}\left(t_{j}, k\right) \geq \frac{1}{2} \theta^{j}
$$

for all $j \in \mathbb{N}$ and living particles, and by convergence the same inequality holds in the limit $n \rightarrow \infty$. In particular we have

$$
\left\langle x\left(t_{j}\right)\right\rangle_{\sigma}^{-} \geq \frac{1}{2} \theta^{j}
$$

On the other hand, it is easy to check that $\langle x\rangle_{\sigma}^{-}$is conserved between particle vanishings, hence we have

$$
\langle x(t)\rangle_{\sigma}^{-} \geq \frac{1}{2} \theta^{j}
$$

whenever $t_{j} \leq t \leq t_{j+1}$. Because of (4.45) we have

$$
t_{j} \sim \theta^{(1-\beta) j}
$$

This means that $t_{j} \leq t \leq t_{j+1}$ implies

$$
\frac{1}{1-\beta} \log _{\theta}(t)-C \leq j \leq \frac{1}{1-\beta} \log _{\theta}(t)+C,
$$

and consequently

$$
\langle x(t)\rangle_{\sigma}^{-} \gtrsim t^{\frac{1}{1-\beta}} .
$$


The upper bound on $\|x\|_{\infty}$ follows in the same way by (4.48). For the case $\beta=1$ the same argument applies, but in this case we have

$$
t_{j}=j T
$$

which leads to

$$
\langle x(t)\rangle_{\sigma}^{-} \gtrsim \theta^{\frac{t}{T}}=\exp (\lambda t) .
$$

The restriction $\lambda \leq 2$ follows from the fact that equation (2.6) for $\beta=1$ implies $\dot{x} \leq$ $2 x$.

The proof of Corollary 2.4 follows easily with a very similar argument:

Proof of Corollary 2.4. Let $\varepsilon>0$. It suffices to consider the case $c=1 / 2$. We apply the same construction as in the proof of Theorem 2.2 (with potentially different $\varepsilon$ in the definition of $\theta$ ) to get an approximate solution $x^{(n)}$, but in the iteration scheme we apply an additional step to $u^{(n, n)}$, satisfying $1 / 2 \leq u^{(n, n)} \leq 1$ according to(4.43). Using Lemma 2.3 with $\varepsilon$ from above yields $\tilde{T}$, only depending on $\beta$ and $\varepsilon$ and a solution $u^{(n, n+1)}$ to the backward equation that satisfies

$$
\left|u^{(n, n+1)}\left(\tau_{n, n}+\tilde{T}, \cdot\right)-\frac{1}{2}\right| \leq \varepsilon
$$

Then the sequence $x^{(n)}$ has the same properties as in the proof of Theorem 2.2 with the addition that the initial data are in an $\varepsilon$-ball around $1 / 2$ for all $n$ by the above inequality, which gives the desired result after sending $n$ to infinity.

\section{Remarks.}

1. For $\beta \in(0,1)$ the achieved growth rate is optimal, because equation (2.6) implies $\dot{x} \leq 2 x^{\beta}$, which can be integrated to obtain $\|x\|_{\infty} \lesssim t^{\frac{1}{1-\beta}}$.

2. The fact that it was possible to choose $\tau_{n, j}-\tau_{n, j-1}=T \theta^{n+1-j}$ in the iteration step was crucial to obtain the desired growth rates. By comparison principle however, the estimate (4.43) also remains valid if we choose a much larger time-span between particle insertions. This means that the above method can be adapted to produce solutions that are unbounded but grow arbitrarily slowly.

3. For convenience we chose $x_{\text {ter }}^{(n)}$ to be constant in the approximation scheme. For the construction however we only used that $\frac{1}{2} \theta^{n} \leq x_{\text {ter }}^{(n)} \leq \theta^{n}$ so that one can use arbitrary sequences satisfying these bounds in our construction scheme to produce solutions with the same coarsening behaviour. 


\section{A Appendix}

Here we we address all technical results that were used in the previous sections and either prove them or give a reference. In the first three parts we discuss aspects of the discrete fast diffusion equation, while the rest of the appendix contains results about parabolic Hölder regularity in the discrete setting.

\section{A.1 The equation $\partial_{t} u=\Delta G_{\beta}(u)$}

We consider the Cauchy problem for the discrete fast diffusion equation

$$
\left\{\begin{array}{l}
\partial_{t} u=\frac{\beta}{|\beta|} \Delta u^{\beta}=\Delta G_{\beta}(u) \quad \text { in }(0, \infty) \times \mathbb{Z}, \\
u(0, \cdot)=u_{0},
\end{array}\right.
$$

with $\beta \in(-\infty, 0) \cup(0,1], u_{0} \in \ell_{+}^{\infty}(\mathbb{Z})$ and

$$
\Delta u=u(k-1)-2 u(k)+u(k+1) .
$$

We are concerned with the long-time existence of classical solutions:

Definition A.1. A function $u:[0, \infty) \rightarrow \ell_{+}^{\infty}(\mathbb{Z})$ is a solution to problem (A.1) if the following conditions are satisfied:

1. $t \mapsto u(t, \cdot)$ is in $C^{0}\left([0, \infty) ; \ell_{+}^{\infty}(\mathbb{Z})\right)$ and $u(0, \cdot)=u_{0}$.

2. For every $k \in \mathbb{Z}$ we have $u(., k) \in C^{1}\left((0, \infty) ; \mathbb{R}_{>0}\right)$ and

$$
\frac{\mathrm{d}}{\mathrm{d} t} u(t, k)=\Delta G_{\beta}(u)(t, k)
$$

for all $k \in \mathbb{Z}$ and $t>0$.

For positive $\beta$ it is not hard to prove the existence of a solution for any kind of initial data, since $G_{\beta}$ is bounded at zero and one has simple a-priori estimates due to the comparison principle (see below). For negative $\beta$ the existence of a sufficiently regular solution for arbitrary data cannot be expected due to $G_{\beta}(x)$ becoming singular at $x=0$. Similar to the result in [HNV16], we have to restrict to initial data which satisfy a certain positivity condition:

Definition A.2. For $u \in \ell_{+}^{\infty}(\mathbb{Z})$ and $d>0$ let

$$
\sigma_{+}(u, k, d)=\inf \{l>k: u(l) \geq d\} .
$$

Then for any $L \in \mathbb{N}$ and $d>0$ we define

$$
\mathcal{P}_{L, d}=\left\{u \in \ell_{+}^{\infty}: \sup _{k \in \mathbb{Z}} \sigma_{+}(u, k, d) \leq L\right\}
$$


In other words, $u \in \mathcal{P}_{L, d}$ means that particles with large mass cannot be very far apart. This is also relevant for the case $\beta>0$ since it allows us to prove certain Harnack-type positivity estimates. We have the following result:

Theorem A.3. Let $\beta \in(-\infty, 0) \cup(0,1]$, and consider initial data $u_{0} \in \mathcal{P}_{L, d}$. Then the following statements hold:

1. If $\beta \in(-\infty, 0) \cup(0,1)$, there exists a positive constant $c=c\left(L, d, \beta,\left\|u_{0}\right\|_{\infty}\right)$ and a solution $u$ to equation (A.1) on $[0, \infty)$ with initial data $u_{0}$ satisfying

$$
u(t, k) \geq c\left(1 \wedge t^{\frac{1}{1-\beta}}\right), \text { for all } k \in \mathbb{Z}
$$

2. If $\beta=1$, the same statement holds with estimate (A.6) replaced by

$$
u(t, k) \geq c \exp (-2 t) I_{L}(2 t)
$$

where $I_{L}(t)$ denotes the $L$-th modified Bessel function of the first kind.

3. Comparison principle: If $c_{1} \leq u_{0} \leq c_{2}$, then $u$ satisfies these bounds for all times.

In this and the next two sections we give a full proof of the above result. The general strategy to prove existence of solutions for equation (2.22) is to use regularization and standard ODE theory. Instead of infinitely many particles with non-negative mass we first consider a periodic $N$-particle ensemble where particles have strictly positive mass. The first important a-priori estimate is the comparison principle:

Lemma A.4 (Finite positive ensemble). Let $\mathbb{T}_{N}$ denote the one-dimensional periodic lattice with $N$ lattice points. Let $u_{0} \in \ell_{+}^{\infty}\left(\mathbb{T}_{N}\right)$ with $0<\delta \leq u_{0} \leq C$. Then there exists a unique solution $u:[0, \infty) \rightarrow \ell_{+}^{\infty}\left(\mathbb{T}_{N}\right)$ of (A.1) with $\delta \leq u(t, \cdot) \leq C$.

Proof. The proof is very similar to Lemma 2 in [EG09] and a standard maximum principle argument. Because $u_{0} \geq \delta$, standard ODE theory gives the existence and uniqueness of a smooth solution $u$ on the time interval $\left[0, t^{*}\right]$ to equation (A.1) with $\delta / 2 \leq u(t,.) \leq 2 C$ for some positive $t^{*}$. For small $\varepsilon>0$ we then consider the solution $u_{\varepsilon}$ of the modified problem

$$
\begin{array}{r}
\partial_{t} u_{\varepsilon}=\Delta G_{\beta}\left(u_{\varepsilon}\right)+\varepsilon, \\
u_{\varepsilon}(0, \cdot)=u_{0},
\end{array}
$$

that exists on the same time interval as $u$ and satisfies the same bounds after possibly making $t^{*}$ smaller. Because $G_{\beta}$ is smooth on $[\delta / 2,2 C]$ we have that $u_{\varepsilon} \rightarrow u$ uniformly on $\left[0, t^{*}\right]$. We claim that $u_{\varepsilon}$ attains its minimum over $\left[0, t^{*}\right] \times \mathbb{T}_{N}$ at $t=0$. If not, there 
exists $t_{0} \in\left(0, t^{*}\right]$ and $k_{1}$ such that $u_{\varepsilon}\left(t_{1}, k_{1}\right)$ is the absolute minimum. Consequently we get

$$
0 \geq \partial_{t} u_{\varepsilon}\left(t_{1}, k_{1}\right)=\Delta G_{\beta}\left(u_{\varepsilon}\right)\left(t_{1}, k_{1}\right)+\varepsilon \geq \varepsilon,
$$

a contradiction. Here we used that $G_{\beta}$ is increasing. Hence $u_{\varepsilon}(t,.) \geq \delta$ for all $t \in\left[0, t^{*}\right]$ and, sending $\varepsilon \rightarrow 0$, the same bound holds for $u$. The same argument for the maximum where $+\varepsilon$ is replaced with $-\varepsilon$ yields that $u \leq C$. Iterating from $t=t^{*}$, we see that the solution can be extended to $[0, \infty)$ and always satisfies the desired bounds.

From this result we can easily pass to the limit as $N \rightarrow \infty$ to obtain solutions for infinite numbers of particles:

Corollary A.5 (Infinite positive ensemble). Let $u_{0} \in \ell_{+}^{\infty}(\mathbb{Z})$ with $0<\delta \leq u_{0} \leq C$. Then there exists a solution $u:[0, \infty) \rightarrow \ell_{+}^{\infty}(\mathbb{Z})$ of (A.1) with $\delta \leq u(t,) \leq$.$C .$

Proof. This is a standard compactness argument. We choose $u_{0}^{(N)}$ to be $N$-periodic such that $u_{0}^{(N)}(k) \rightarrow u_{0}(k)$ for each $k \in \mathbb{Z}$. Let $u^{(N)}$ be the corresponding solutions from the above lemma. Then due to the a-priori bounds $\delta \leq u^{(N)} \leq C$ and equation (A.1) we have

$$
\left|\frac{\mathrm{d}}{\mathrm{d} t} u^{(N)}\right|=\left|\Delta G_{\beta}\left(u^{(N)}\right)\right| \leq K(\delta, C),
$$

hence the solutions are uniformly Lipschitz continuous. Applying the Arzela-Ascoli Theorem and a diagonal argument we can extract a convergent subsequence (not relabeled) such that $u^{(N)}(\cdot, k) \rightarrow u(\cdot, k)$ for some uniformly on compact time intervals, where $u(\cdot, k) \in C^{0}\left([0, \infty)\right.$. In particular $u$ satisfies the same bounds as $u^{(N)}$. Integrating (A.1) in time and passing to the limit (which is possible due to the a-priori bounds) then yields

$$
u(t, k)=u_{0}(k)+\int_{0}^{t} \Delta_{\sigma} G_{\beta}(u)(s, k) \mathrm{d} s .
$$

This in turn shows that $u(., k)$ is continuously differentiable and solves (A.1) pointwise. Again, the bounds on $u$ yield Lipschitz continuity in $\ell_{+}^{\infty}(\mathbb{Z})$.

For our purpose, we need the existence of solutions in particular for initial data with mass zero particles. The general strategy is to approximate the initial data by regularized data via

$$
u_{0, \delta}=u_{0} \vee \delta .
$$

The above existence result then yields long-time solutions $u_{\delta}$ with initial data $u_{0, \delta}$. In the case $\beta>0$ one can pass to the limit $\delta \rightarrow 0$ in the same manner as above, since $G_{\beta}$ is bounded at zero, yielding a general existence result: 
Corollary A.6 (Existence for positive $\beta$ ). Let $\beta \in(0,1]$ and $u_{0} \in \ell_{+}^{\infty}(\mathbb{Z})$. Then there exists a solution $u:[0, \infty) \rightarrow \ell_{+}^{\infty}(\mathbb{Z})$ of (A.1).

Alternatively it is likely possible to prove this result directly via an infinite dimensional fixed-point method. Since we need Corollary (A.5) for the case $\beta<0$ anyway, the above method is the fastest for our purpose. In the next section we deal with the negative $\beta$ case, including existence and the positivity estimate (A.6). Then we prove the positivity estimate for positive $\beta$, completing the proof of Theorem A.3.

\section{A.2 Existence of solutions for $\beta<0$}

In the following we always assume $\beta<0$. The key idea to prove existence of solutions is to exploit the fact that regions which are enclosed by large particles (called traps) are screened from the rest of the particles, very similar to HNV16. One important difference however is the fact that the backward equation does not yield a-priori estimates on the persistence of traps. We make the following definition:

Definition A.7. We say that a solution $u$ to equation (A.1) with initial data $u_{0} \in \mathcal{P}_{L, d}$ has the persistence property on $[0, T]$ if $u(0, k) \geq d$ implies $u(t, k) \geq \frac{d}{2}$ for all $t \in[0, T]$.

By making use of the theory for the coarsening equation developed in [HNV16] we have the following result concerning Hölder regularity:

Lemma A.8. There exist constants $T^{\prime}=T^{\prime}(\beta, d)$ and $C=C(\beta, L)>0$ such that the following holds: If a solution $u$ to equation (A.1) with initial data $u_{0} \in \mathcal{P}_{L, d}$ has the persistence property on $[0, T]$ and $T \leq T^{\prime}$, then

$$
\left|u\left(t_{2}, k\right)-u\left(t_{1}, k\right)\right| \leq C\left|t_{2}-t_{1}\right|^{\frac{1}{1-\beta}},
$$

for all $t_{2}, t_{1} \in[0, T]$ and $k \in \mathbb{Z}$.

Proof. We consider the time reversed function

$$
x(s, k)=u(T-s, k),
$$

then $x$ is a solution to the coarsening equation for $0 \leq s \leq T$ that satisfies $x(0,.) \in \mathcal{P}_{L, \frac{d}{2}}$. Applying Lemma 3.3 from [HNV16] (if $T \leq T^{*}\left(\beta, \frac{d}{2}\right)=: T^{\prime}$ ) yields the desired Hölder continuity for $x$, and thus also for $u$.

From this result we derive the first a-priori estimate:

Lemma A.9. Let $u_{0, \delta}$ be as above and let $u_{\delta}$ be the corresponding solution of equation (A.1), which exists by Lemma A.5. Then there exists $T=T(L, d)>0$ such that $u_{\delta}$ has the persistence property on $[0, T]$. 
Proof. First we note that because the solution satisfies $u_{\delta} \geq \delta$ for all times then we have the Lipschitz estimate

$$
\left|\partial_{t} u_{\delta}\right| \leq 4 \delta^{\beta}
$$

This means that $u_{\delta}(0, k) \geq d$ implies $u_{\delta}(t, k) \geq \frac{d}{2}$ for $0 \leq t \leq t_{0}$, where

$$
t_{0}=\frac{d}{8 \delta^{\beta}}
$$

Let $T$ be the largest time such that $u_{\delta}(0, k) \geq d$ implies $u_{\delta}(t, k) \geq \frac{d}{2}$ on $[0, T]$. By the above considerations we already know that $T>0$. If $T \leq T^{\prime}(\beta, d)$ we can apply Lemma A.8 and get

$$
u_{\delta}(t, k) \geq u_{\delta}(0, k)-C t^{\frac{1}{1-\beta}}
$$

If $u_{\delta}(0, k) \geq d$, this implies $u_{\delta}(T, k) \geq d-C T^{\frac{1}{1-\beta}}$. On the other hand, by the definition of $T$ there exists such a $k$ with $u_{\delta}(T, k) \leq \frac{3 d}{4}$, hence

$$
\frac{3 d}{4} \geq d-C(L) T^{\frac{1}{1-\beta}}
$$

which gives a lower bound for $T$ in terms of $L$ and $d$.

The next a-priori estimate is crucial to get uniform Hölder bounds on $u_{\delta}$, as well as integral bounds which are needed to pass to the limit.

Lemma A.10. Let $u_{\delta}$ be as above. Then there exists $c=c(\beta, L, d)>0$ and $t^{*}=$ $t^{*}(\beta, L, d)>0$ such that

$$
u_{\delta}(t, \cdot) \geq c t^{\frac{1}{1-\beta}}
$$

for $0 \leq t \leq t^{*}$.

Proof. We apply a very similar argument as in the proof of Lemma 3.5 in [HNV16]. If the statement is false, there exist sequences $u^{(n)}, t_{n} \rightarrow 0, \delta_{n} \rightarrow 0$ and $k_{n} \in \mathbb{Z}$ such that

$$
u_{\delta_{n}}^{(n)}\left(t_{n}, k_{n}\right) \leq \frac{1}{n} t_{n}^{\frac{1}{1-\beta}} .
$$

By translation invariance we can assume that $k_{n}=k_{0}$ is constant. We rescale and define 


$$
v_{n}(s, k)=t_{n}^{\frac{1}{\beta-1}} u_{\delta_{n}}^{(n)}\left(t_{n} s, k\right) .
$$

Then $v_{n}$ is a solution to equation (A.1) with $v_{n}\left(1, k_{0}\right) \rightarrow 0$. Additionally we have $v_{n}(0,.) \in \mathcal{P}_{L, d}$ and $v_{n}$ satisfies the persistence property on $[0,1]$ for large $n$ by Lemma A.9. Since $t_{n}^{\frac{1}{\beta-1}} d \rightarrow \infty$ and $T^{\prime} \rightarrow \infty$ as $d \rightarrow \infty$ we also have that $v_{n}$ is uniformly Hölder continuous by Lemma A.8, Let $B$ be the largest set of consecutive indices containing $k_{0}$ such that

$$
\liminf _{n \rightarrow \infty} v_{n}(1, k)>0
$$

for $k \in B$. Observe that we have $|B| \leq L$ due to $t_{n}^{\frac{1}{\beta-1}} d \rightarrow \infty$ and the persistence property. Let $l_{-}, l_{+}$be the nearest particle index to the left, respectively to the right of $B$. We restrict to a subsequence such that $v_{n}(1, k) \rightarrow 0$ for $k \in B$ and $v_{n}\left(1, l_{ \pm}\right) \geq \lambda>0$. If we define the local mass $M_{n}(s)$ as

$$
M_{n}(s)=\sum_{k \in B} v_{n}(s, k)
$$

then an elementary calculation gives

$$
\frac{\mathrm{d}}{\mathrm{d} s} M_{n}(s)=v_{n}^{\beta}\left(s, l_{-}+1\right)-v_{n}^{\beta}\left(s, l_{-}\right)+v_{n}^{\beta}\left(s, l_{+}-1\right)-v_{n}^{\beta}\left(s, l_{+}\right) .
$$

Due to uniform Hölder continuity and particles in $B$ going to zero there exists $\varepsilon>0$ such that

$$
v_{n}^{\beta}\left(s, l_{ \pm}\right) \leq \frac{1}{2} v_{n}^{\beta}\left(s, l_{ \pm} \mp 1\right),
$$

for $s \in[1-\varepsilon, 1]$ and large enough $n$. Using equation (A.25) on this time interval we obtain

$$
2 \frac{\mathrm{d}}{\mathrm{d} s} M_{n}(s) \geq v_{n}^{\beta}\left(s, l_{-}+1\right)+v_{n}^{\beta}\left(s, l_{+}-1\right) \geq M_{n}^{\beta}(s),
$$

which, after integrating from $1-\varepsilon$ to 1 yields

$$
M_{n}(1) \geq \tilde{\varepsilon}>0
$$

which gives a contradiction after sending $n$ to infinity. 
This result gives us the a-priori estimates we need to pass to the limit:

Corollary A.11 (Hölder continuity). Let $u_{\delta}$ and $t^{*}$ be as above. Then there exists $C=C(\beta, L, d)$ such that

$$
\left|u_{\delta}\left(t_{2}, k\right)-u_{\delta}\left(t_{1}, k\right)\right| \leq C\left|t_{2}-t_{1}\right|^{\frac{1}{1-\beta}},
$$

for all $t_{2}, t_{1} \in\left[0, t^{*}\right]$ and $k \in \mathbb{Z}$.

Proof. Let $t_{2}, t_{1} \in\left[0, t^{*}\right], t_{2}>t_{1}$. We integrate (A.1) in time and estimate

$$
\begin{aligned}
\left|u\left(t_{2}, k\right)-u\left(t_{1}, k\right)\right| & \leq \int_{0}^{t}\left|\Delta G\left(u_{\delta}\right)(s, k)\right| \mathrm{d} s \lesssim \int_{t_{1}}^{t_{2}} s^{\frac{\beta}{1-\beta}} \mathrm{d} s \\
& \sim t_{2}^{\frac{1}{1-\beta}}-t_{1}^{\frac{1}{1-\beta}} \leq\left(t_{2}-t_{1}\right)^{\frac{1}{1-\beta}},
\end{aligned}
$$

where we used Lemma A.10 to estimate $\left|\Delta G_{\beta}\left(u_{\delta}\right)\right|$.

With these preparations we can prove the first statement of Theorem A.3 for negative $\beta$ :

Proof of existence and positivity bound for $\beta<0$. Let $u_{0, \delta}$ and $u_{\delta}$ as above. By Corollary A.11 and the Arzela-Ascoli Theorem there exists a subsequence $\delta \rightarrow 0$ such that $u_{\delta} \rightarrow u$ uniformly on $\left[0, t^{*}\right]$. Moreover, by Lemma A.10 we have

$$
u_{\delta}^{\beta}(t, k) \leq c^{\beta} t^{\frac{\beta}{1-\beta}}
$$

which implies $u_{\delta}^{\beta} \rightarrow u^{\beta}$ in $L^{1}\left(\left[0, t^{*}\right]\right)$. Using this we can pass to the limit in the integral equation

$$
u_{\delta}(t, k)=u_{0, \delta}(k)-\int_{0}^{t} \Delta G\left(u_{\delta}\right)(s, k) \mathrm{d} s
$$

showing that $u$ is a solution to the backward equation with initial data $u_{0}$ on $\left[0, t^{*}\right]$. Since the lower bound from Lemma A.10 also holds in the limit, we can extend the solution from $t^{*}$ to arbitrary times via comparison principle, which also changes the lower bound to $\sim 1 \wedge t^{\frac{1}{1-\beta}}$ for large times. 


\section{A.3 Harnack-type inequality for $0<\beta \leq 1$}

In the previous part Lemma A.10 was crucial to prove existence of a solution. The result of the lemma, together with the positivity condition $\mathcal{P}_{L, d}$ can be interpreted as a Harnack-type inequality, see [BV06]. For $0<\beta<1$ a similar result holds, the equation however behaves differently and the indirect proof does not work here. We will pursue another approach and show the inequality directly with explicit constants, handling the case $\beta=1$ separately. The key observation is that a large particle next to a small particle will always induce growth on the small particle, despite the size of the other neighbour of the small particle. This decouples the equation in a sense and we only need to study the local problem:

Lemma A.12 (Local Problem). Let $0<\beta<1$ and $T>0$. Consider two functions $F \in C^{0}([0, T] ;[0, \infty))$ and $u \in C^{1}([0, T] ;[0, \infty))$ which satisfy

$$
\begin{aligned}
F(t) & \geq c t^{\frac{\beta}{1-\beta}}, \\
\dot{u}(t) & \geq F(t)-2 u^{\beta}(t) .
\end{aligned}
$$

on $[0, T]$. Then $u$ satisfies

$$
u(t) \geq \eta_{1}(c) t^{\frac{1}{1-\beta}}
$$

on the interval $[0, T]$, where $\eta_{1}$ is a positive strictly increasing function which depends only on $\beta$.

Proof. We define the rescaled function

$$
v(t)=t^{\frac{1}{\beta-1}} u(t)
$$

on the half-open interval $(0, T]$. Then it suffices to show that $v$ is bounded from below by $\eta_{1}(c)$. We calculate

$$
\begin{aligned}
t \dot{v}(t) & =t\left(t^{\frac{1}{\beta-1}} \dot{u}(t)+\frac{1}{\beta-1} t^{\frac{1}{\beta-1}-1} u(t)\right) \\
& \geq t\left(t^{\frac{1}{\beta-1}} F(t)-2 t^{\frac{1}{\beta-1}} u^{\beta}(t)+\frac{1}{\beta-1} t^{\frac{1}{\beta-1}-1} u(t)\right) \\
& =t^{\frac{\beta}{\beta-1}} F(t)-2 v^{\beta}(t)-\frac{1}{1-\beta} v(t) \\
& =: t^{\frac{\beta}{\beta-1}} F(t)-\theta(v(t))
\end{aligned}
$$

in particular the assumption on $F$ implies that 


$$
t \dot{v}(t) \geq c-\theta(v(t)) .
$$

Since the function $\theta$ is strictly increasing on $[0, \infty)$ we can define the inverse function $\eta_{1}=\theta^{-1}$, which is also strictly increasing. We claim that $v \geq \eta_{1}(c)$ on $(0, T]$. If this is not true, there is $\varepsilon>0$ and $t^{*} \in(0, T]$ such that $v\left(t^{*}\right) \leq \eta_{1}(c)-\varepsilon$. In particular we have

$$
c-\theta\left(v\left(t^{*}\right)\right) \geq \tilde{\varepsilon}>0,
$$

for some $\tilde{\varepsilon}>0$. But then the differential inequality (A.42) implies that $v(t) \leq \eta_{1}(c)-\varepsilon$, and hence $c-\theta(v(t)) \geq \tilde{\varepsilon}$ for all $t \in\left(0, t^{*}\right]$. Dividing by $t$ and integrating (A.42) in time gives

$$
v\left(t^{*}\right)-v(t) \geq \varepsilon \log \left(\frac{t^{*}}{t}\right),
$$

for all $0<t \leq t^{*}$. Sending $t$ to zero then gives a contradiction.

The above lemma enables us to prove a Harnack type inequality:

Lemma A.13 (Harnack type inequality). Let $u$ be a solution to equation (A.1) with $0<\beta<1$ and initial data $0 \leq u_{0} \leq 1$. Then we have

$$
u(t, k) \geq \eta(|k-l|)(t-s)^{\frac{1}{1-\beta}},
$$

for all $k, l \in \mathbb{Z}$ and $0 \leq t-s \leq t^{*}(u(s, l))$. The function $\eta$ is strictly positive and the function $t^{*}$ is non-negative, strictly increasing with $t^{*}(u)=0$ iff $u=0$. Furthermore, both functions depend only on $\beta$.

Proof. Due to translation invariance in space and time it suffices to consider the case $s=0$ and $l=0$. We will make an iterative argument, using Lemma A.12 in each step. First we note that due to $u_{0} \leq 1$ and the comparison principle we have the Lipschitz estimate

$$
|\dot{u}| \leq 4,
$$

in particular

$$
u(t, 0) \geq u_{0}(0)-4 t .
$$

The case $u_{0}(0)=0$ is trivial. If $u_{0}(0)>0$, the Lipschitz estimate implies 


$$
u(t, 0) \geq t^{\frac{1}{1-\beta}},
$$

whenever $u_{0}(0)-4 t-t^{\frac{1}{1-\beta}}>0$. If we set $f(t)=4 t+t^{\frac{1}{1-\beta}}$ then $t^{*}$ is defined as the inverse of $f$. Thus the above lower bound holds for $0 \leq t \leq t^{*}\left(u_{0}(0)\right)$. For $u(t, 1)$ we have

$$
\begin{aligned}
\dot{u}(t, 1) & =u^{\beta}(t, 0)-2 u^{\beta}(t, 1)+u^{\beta}(t, 2) \\
& \geq u^{\beta}(t, 0)-2 u^{\beta}(t, 1) .
\end{aligned}
$$

This means that $u^{\beta}(t, 0)$ and $u(t, 1)$ satisfy the assumptions of Lemma A.12 with $T=$ $t^{*}\left(u_{0}(0)\right)$ and $c=1$. Thus we have

$$
u(t, 1) \geq \eta_{1}(1) t^{\frac{1}{1-\beta}}
$$

on $\left[0, t^{*}\left(u_{0}(0)\right)\right]$. Now we can successively apply the same argument to the pairs of functions $\left(u^{\beta}(t, 1), u(t, 2)\right), \ldots,\left(u^{\beta}(t, k-1), u(t, k)\right)$, for $k \in \mathbb{N}$. The argument for $-k$ is the same. Then the desired inequality follows with the function $\eta=\eta(r)(r \in \mathbb{N})$ defined as

$$
\begin{aligned}
& \eta(r)=\eta_{1}^{(r)}(1), \\
& \eta(0)=1
\end{aligned}
$$

where $\eta_{1}^{(r)}$ means that $\eta$ is $r$ times composed with itself.

Lemma A.14. Let $u$ be a solution to the constant coefficient linear equation equation (A.1) with $\beta=1$. Then for every $k \in \mathbb{Z}$ and $N \in \mathbb{N}$ we have

$$
u(t, k) \geq M\left(u_{0}, k, N\right) \exp (-2 t) I_{N}(2 t),
$$

where $I_{k}(t)$ denotes the $k$-th modified Bessel function of the first kind and

$$
M\left(u_{0}, k, N\right)=\sum_{l=-N}^{N} u_{0}(k-l)
$$

denotes the local initial mass. 
Proof. In the linear constant-coefficient case we can give an explicit formula by Fourieranalysis: We write

$$
\hat{u}(t, \theta)=\sum_{k=-\infty}^{k=+\infty} u(t, k) \exp (-i k \theta),
$$

taking the time derivative on both sides and using the equation then yields

$$
\begin{aligned}
\partial_{t} \hat{u}(t, \theta) & =\exp (-i \theta) \sum_{k=-\infty}^{k=+\infty} u(t, k) \exp (-i k \theta) \\
& -2 \sum_{k=-\infty}^{k=+\infty} u(t, k) \exp (-i k \theta) \\
& +\exp (i \theta) \sum_{k=-\infty}^{k=+\infty} u(t, k) \exp (-i k \theta) \\
& =2(\cos (\theta)-1) \hat{u}(t, \theta) .
\end{aligned}
$$

We solve this ODE in $t$ with initial data $\hat{f}$ to obtain

$$
\hat{u}(t, \theta)=\hat{f}(\theta) \exp (2 t(\cos (\theta)-1)),
$$

which gives the discrete heat kernel

$$
\begin{aligned}
\phi(t, k) & =\exp (-2 t) \frac{1}{2 \pi} \int_{-\pi}^{\pi} \exp (2 t \cos (\theta)-i k \theta) \mathrm{d} \theta \\
& =\exp (-2 t) \frac{1}{\pi} \int_{0}^{\pi} \exp (2 t \cos (\theta)) \cos (k \theta) \mathrm{d} \theta \\
& =\exp (-2 t) I_{k}(2 t),
\end{aligned}
$$

where $I_{k}$ is the $k$ th modified Bessel function of the first kind. Then the desired inequality follows directly by the standard representation

$$
u(t, k)=\sum_{l \in Z} u_{0}(k-l) \phi(t, l),
$$

the fact that $\phi$ is decreasing in the second argument and the obvious estimate.

We summarize the findings of this section and prove the remaining statements of Theorem A.3. 
Proof of positivity estimate for $0<\beta \leq 1$. First we consider the case $\beta \neq 1$. It suffices to consider the case $u_{0} \leq 1$ by scaling (this means that for general data the constants get an additional dependence on $\left.\left\|u_{0}\right\|_{\infty}\right)$. Because $u_{0} \in \mathcal{P}_{L, d}$, for every $k \in \mathbb{Z}$ there exists $k^{\prime}$ with $u_{0}\left(k^{\prime}\right) \geq d$ and $\left|k-k^{\prime}\right| \leq L$. Then Lemma A.13 with $s=0$ and $l=k^{\prime}$ yields

$$
u(t, k) \geq \min _{j=1, . ., L} \eta(j) t^{\frac{1}{1-\beta}},
$$

for $0 \leq t \leq t^{*}(d)$ because $t^{*}$ is monotone. For $\beta=1$ we note that $u_{0} \in \mathcal{P}_{L, d}$ implies that $M(u, k, L) \geq 2 d$, since there are at least two terms in the sum that are greater than or equal to $d$ by definition of $\mathcal{P}_{L, d}$. Then the statement follows directly from Lemma A.14.

\section{A.4 Nash-Aronson estimates and Hölder continuity}

For $u=u(k) \in \ell^{\infty}(\mathbb{Z})$ we define the forward and backward difference operators

$$
\begin{aligned}
& \partial^{+} u(k)=u(k+1)-u(k) \\
& \partial^{-} u(k)=u(k)-u(k-1) .
\end{aligned}
$$

For $a=a(t, k)$ with $0<c_{1} \leq a \leq c_{2}$ and $a(., k) \in C^{0}([0, \infty))$ we consider the discrete analogue to a parabolic evolution equation in divergence form:

$$
\left\{\begin{array}{l}
\partial_{t} u=\partial^{-}\left(a \partial^{+} u\right)=: \mathcal{L}(t) u \\
u(0, .)=u_{0} .
\end{array}\right.
$$

We denote by $\phi(t, k, s, l)$ the fundamental solution to (A.69), in other words, $\phi(., ., s, l)$ is the solution to the above equation starting at time $s$ with initial data $\phi_{0}(k)=\delta_{k l}$. Since $\mathcal{L}(t)$ is a bounded operator from $\ell^{2}(\mathbb{Z})$ to $\ell^{2}(\mathbb{Z}), \phi$ can be written as

$$
\phi(t, k, s, l)=\left\langle\exp \left(\int_{s}^{t} \mathcal{L}(r) \mathrm{d} r\right) \delta_{l}, \delta_{k}\right\rangle,
$$

where $\delta_{k}$ are the canonical basis vectors in $\ell^{2}(\mathbb{Z})$. The general solution starting at time $t=s$ to (4.22) is then given by

$$
u(t, k)=\sum_{l \in \mathbb{Z}^{d}} \phi(t, k, s, l) u_{0}(l) .
$$

We also define the reduced fundamental solution

$$
\psi(t, k)=\phi(t, k, 0,0)=\phi(t, 0,0, k),
$$


and the corresponding "macroscopic" rescaled function

$$
\begin{aligned}
& U: \mathbb{R} \rightarrow[0,+\infty) \\
& U(t, \xi)=t^{\frac{1}{2}} \psi\left(t,\left\lfloor t^{\frac{1}{2}} \xi\right\rfloor\right) .
\end{aligned}
$$

We have the following Nash-Aronson estimates on the fundamental solution:

Theorem A.15. There exist constants $t_{0}>0, C>0$ and $\alpha>0$, depending only on the bounds on a, such that the following statements hold:

- Aronson estimate:

$$
\psi(t, k) \leq \frac{C}{1 \vee t^{\frac{1}{2}}} \exp \left(-\frac{|k|}{1 \vee t^{\frac{1}{2}}}\right),
$$

for every $k \in \mathbb{Z}$ and $t \geq 0$.

- Nash continuity estimate:

$$
|\psi(t, k)-\psi(t, l)| \leq \frac{C}{t^{\frac{1}{2}}}\left(\frac{|k-l|}{t^{\frac{1}{2}}}\right)^{\alpha},
$$

for every $k, l \in \mathbb{Z}$ and $t \geq 0$.

Proof. Here we cite the results from Appendix B of [GOS01. Inequality (A.75) is precisely the statement of Proposition B.3. For the second inequality (A.76) we first note that (A.75) implies $\left|\psi_{1 / 2}\right| \lesssim t^{-\frac{1}{2}}$. Then the desired estimate at a time $t^{*}$ follows from Proposition B.6 applied at $t=s=t^{*} / 2$ with $f=\psi\left(t^{*} / 2,.\right)$ and the semigroup property.

These estimates have important consequences for the function $U$. Inequality A.75 implies that

$$
U(t, \xi) \leq \Phi(\xi)
$$

for some integrable function $\Phi$. In particular the function family $U(t,$.$) are tight proba-$ bility measures. On the other hand, the estimate (A.76) implies that the function $U(t,$.$) ,$ which is a step-function by definition, becomes Hölder continuous in the following sense:

Definition A.16 (Approximate Hölder Continuity). Let $\left\{f_{n}\right\} \subset L^{\infty}(\mathbb{R})$ be a sequence of functions. Then $\left\{f_{n}\right\}$ is said to be approximately Hölder continuous with exponent $\alpha \in(0,1]$ if for every $\varepsilon>0$ there exists $n=n(\varepsilon)$ such that $|x-y| \geq \varepsilon$ implies

$$
\left|f_{n}(x)-f_{n}(y)\right| \leq C|x-y|^{\alpha},
$$

for $n \geq n(\varepsilon)$ and a universal positive constant $C$. 
The important observation is that Hölder continuity on the discrete microscopic level implies approximate Hölder continuity on the macroscopic scale:

Lemma A.17. The function $U(t,$.$) is approximately Hölder continuous as t \rightarrow \infty$. Furthermore, the constants $C, \alpha$ and $t=t(\varepsilon)$ only depend on the bounds of the coefficient a in A.69).

Proof. By the estimate (A.76) from Theorem A.15 we calculate

$$
|U(t, \xi)-U(t, \eta)| \lesssim\left(\frac{\left\lfloor\left\lfloor t^{\frac{1}{2}} \xi\right\rfloor-\left\lfloor t^{\frac{1}{2}} \eta\right\rfloor \mid\right.}{t^{\frac{1}{2}}}\right)^{\alpha}
$$

Since

$$
\frac{\left\lfloor\left\lfloor t^{\frac{1}{2}} \xi\right\rfloor-\left\lfloor t^{\frac{1}{2}} \eta\right\rfloor \mid\right.}{t^{\frac{1}{2}}}=|\xi-\eta|+\mathcal{O}\left(t^{-\frac{1}{2}}\right),
$$

we get the desired estimate for $|\xi-\eta| \gtrsim t^{-\frac{1}{2}}$.

The next result is of crucial importance for the main result of the paper. Denote by $\mathcal{T}_{\delta}(\mathbb{R})$ the set of step-functions with step-width at least $\delta$. Then we have:

Lemma A.18. Let $\left(f_{n}\right) \subset L^{1}(\mathbb{R})$ be tight and approximately Hölder continuous. Then for every $\varepsilon>0$ there exists $n_{0}$ and $\delta>0$, such that for every $f_{n}$ with $n \geq n_{0}$ there exists $\chi \in \mathcal{T}_{\delta}(\mathbb{R})$ with

$$
\left\|f_{n}-\chi\right\|_{L^{1}(\mathbb{R})} \leq \varepsilon
$$

Proof. Let $\varepsilon>0$. Because $\left(f_{n}\right)$ is tight in $L^{1}$ there exists $R>0$ such that

$$
\int_{|x| \geq R}\left|f_{n}(x)\right| \mathrm{d} x \leq \varepsilon
$$

hence it suffices to approximate $\left(f_{n}\right)$ in $L^{\infty}$. By approximate Hölder continuity there exists $n_{0}$ such that

$$
\left|f_{n}(x)-f_{n}(y)\right| \leq C|x-y|^{\alpha},
$$

for $n \geq n_{0}$ and $|x-y| \geq R^{-\frac{1}{\alpha}} \varepsilon$. This means that the piecewise-constant interpolation $\chi$ of $f_{n}$ with step-width $R^{-\frac{1}{\alpha}} \varepsilon$ approximates $f_{n}$ uniformly up to an error of $R^{-1} \varepsilon^{\alpha}$, hence

$$
\left\|f_{n}-\chi\right\|_{L^{1}} \lesssim \varepsilon+\varepsilon^{\alpha}
$$


Combining the last two lemmas we obtain the following corollary, which is used in the proof of the main result:

Corollary A.19. For every $\varepsilon>0$ there exists $T>0$ and $\delta>0$, such that for every $U(t,$.$) with t \geq T$ there exists $\chi \in \mathcal{T}_{\delta}(\mathbb{R})$ with

$$
\|U(t, .)-\chi\|_{L^{1}(\mathbb{R})} \leq \varepsilon .
$$

Furthermore, $T$ and $\delta$ only depend on $\varepsilon$ and the bounds on a.

Proof. Approximate Hölder continuity was already established, while tightness in $L^{1}$ follows from the estimate (A.75) of Theorem A.15. The dependence of the constants is easily checked revisiting the proofs of the previous two lemmas.

\section{Acknowledgement}

The author would like to thank Barbara Niethammer and Juan J. L. Velázquez for inspiration, helpful discussions and proofreading. This work was supported by the German Research Foundation through the CRC 1060 The Mathematics of Emergent Effects.

\section{References}

[BV06] Matteo Bonforte and Juan Luis Vazquez. Global positivity estimates and Harnack inequalities for the fast diffusion equation. Journal of Functional Analysis, 240(2):399-428, 2006.

[EG09] Selim Esedoglu and John B. Greer. Upper bounds on the coarsening rate of discrete, ill-posed nonlinear diffusion equations. Communications on Pure and Applied Mathematics, 62(1):57-81, 2009.

[ES08] Selim Esedoglu and Dejan Slepčev. Refined upper bounds on the coarsening rate of discrete, ill-posed diffusion equations. Nonlinearity, 21(12):2759, 2008.

[GOS01] Giambattista Giacomin, Stefano Olla, and Herbert Spohn. Equilibrium Fluctuations for $\nabla_{\varphi}$ Interface Model. Ann. Probab., 29(3):1138-1172, 072001.

[GW03] K. B. Glasner and T. P. Witelski. Coarsening dynamics of dewetting films. Phys. Rev. E, 67:016302, Jan 2003.

[GW05] K.B. Glasner and T.P. Witelski. Collision versus collapse of droplets in coarsening of dewetting thin films. Physica D: Nonlinear Phenomena, 209(1):80-104, 2005. Non-linear Dynamics of Thin Films and Fluid Interfaces.

[HK02] E. K. O. Hellén and J. Krug. Coarsening of sand ripples in mass transfer models. Phys. Rev. E, 66:011304, Jul 2002. 
[HNO04] Reiner Henseler, Barbara Niethammer, and Felix Otto. A Reduced Model for Simulating Grain Growth. In Pierluigi Colli, Claudio Verdi, and Augusto Visintin, editors, Free Boundary Problems, pages 177-187, Basel, 2004. Birkhäuser Basel.

[HNV16] Michael Helmers, Barbara Niethammer, and Juan J. L. Velázquez. Mathematical Analysis of a Coarsening Model with Local Interactions. J. Nonlinear Science, 26(5):1227-1291, 2016.

[KO02] Robert V. Kohn and Felix Otto. Upper Bounds on Coarsening Rates. Communications in Mathematical Physics, 229(3):375-395, Sep 2002.

[Nas58] J. Nash. Continuity of Solutions of Parabolic and Elliptic Equations. American Journal of Mathematics, 80(4):931-954, 1958.

[vvL01] Roger M. van der Meer, J.P. van der Weele, and Detlef Lohse. Bifurcation diagram for compartmentalized granular gases. Physical review E: covering statistical, nonlinear, biological, and soft matter physics, 63(061304):0613041-061304-9, 2001. 